\title{
The geodesic approximation for lump dynamics and coercivity of the Hessian for harmonic maps
}

\author{
M. Haskins* \\ Department of Mathematics, Johns Hopkins University \\ Baltimore, MD 21218, U.S.A. \\ J.M. Speight ${ }^{\dagger}$ \\ Department of Pure Mathematics, University of Leeds \\ Leeds LS2 9JT, England
}

\begin{abstract}
The most fruitful approach to studying low energy soliton dynamics in field theories of Bogomol'nyi type is the geodesic approximation of Manton. In the case of vortices and monopoles, Stuart has obtained rigorous estimates of the errors in this approximation, and hence proved that it is valid in the low speed regime. His method employs energy estimates which rely on a key coercivity property of the Hessian of the energy functional of the theory under consideration. In this paper we prove an analogous coercivity property for the Hessian of the energy functional of a general sigma model with compact Kähler domain and target. We go on to prove a continuity property for our result, and show that, for the $\mathbb{C} P^{1}$ model on $S^{2}$, the Hessian fails to be globally coercive in the degree 1 sector. We present numerical evidence which suggests that the Hessian is globally coercive in a certain equivariance class of the degree $n$ sector for $n \geq 2$. We also prove that, within the geodesic approximation, a single $\mathbb{C} P^{1}$ lump moving on $S^{2}$ does not generically travel on a great circle.
\end{abstract}

\section{Introduction}

Many field theories arising naturally in theoretical high energy physics may be said to be of Bogomol'nyi type. For such theories there is a topological lower bound on the energy of field configurations, and this bound is attained only by solutions of a first order "selfduality" equation, the so-called solitons of the theory. The solitons are stable by virtue of their energy-minimizing property, and are generically spatially localized lumps of energy with strongly particle-like characteristics. When static they exert no net force on one another, so

${ }^{*}$ E-mail: mhaskin@math.jhu.edu

${ }^{\dagger}$ E-mail: j.m.speight@leeds.ac.uk 
the structure of the space of static multisoliton solutions is rather rich. Examples within the context of gauge theory are given by the Yang-Mills-Higgs and abelian-Higgs models, whose solitons are called monopoles and vortices respectively. In both these cases, the static models are very well understood and the structure of $\mathrm{M}_{n}$, the moduli space of static $n$-soliton solutions, is known in great detail. For monopoles, in particular, the static system is integrable and there are several constructions which generate exact solutions of various degrees of explicitness. Once one introduces time dependence, however, things get much more difficult. No Bogomol'nyi type field theory (indeed, no Lorentz invariant field theory) in more than $(1+1)$ dimensions is integrable, and the construction of nontrivial exact time-dependent solutions seems impossible.

How then is one to understand the dynamics of moving solitons in these models? The most fruitful approach has turned out to be the geodesic approximation of Manton [11]. Here one argues on physical grounds that the solution of any initial value problem in the $n$-soliton sector whose initial field is a static solution, and whose initial kinetic energy is small, should be forced to stay close to $\mathrm{M}_{n}$ by energy conservation. Manton suggested that the dynamics should be well approximated by a solution of the reduced variational problem where the field configuration is constrained to lie on $\mathrm{M}_{n}$ at all times. This reduced dynamics turns out to be geodesic flow on $\mathrm{M}_{n}$ with respect to a natural metric called the $L^{2}$ metric, so one has the appealing suggestion that low energy soliton dynamics in Bogomol'nyi type theories may be understood by studying the Riemannian geometry of their moduli spaces. This is still a highly nontrivial problem, and it was some time before the $L^{2}$ metrics on the 2-monopole and 2-vortex moduli spaces were well understood [2, 17]. Since first being proposed, the method of Manton has been extended to deal with dynamical issues other than classical multisoliton scattering. Quantum soliton states [5] are thought to be well approximated by eigenstates of the Laplacian on $\mathrm{M}_{n}$, for example, and the thermodynamics of soliton gases [12] has been analyzed in terms of the geometry of $\mathrm{M}_{n}$.

Not all Bogomol'nyi type field theories are gauge theories. Another class is given by nonlinear sigma models with Kähler target space, for example the $\mathbb{C} P^{N}$ models. These models are nonlinear in the most fundamental way: the field takes values in a space with no linear structure. However they have many features in common with the gauge theories mentioned above. There is again a topological lower bound on energy, attained only by solutions of a first order partial differential equation, namely the \pm -holomorphic maps - the Cauchy-Riemann conditions play the role of the self-duality equation. The static model is integrable and the structure of $\mathrm{M}_{n}$ is again well understood. The solitons in this case are usually called "lumps."

Given the similarities between lumps and their gauge theoretic counterparts, it was natural for Ward to suggest, in the specific context of the $\mathbb{C} P^{1}$ model on the plane, that the geodesic approximation should be applicable to classical lump dynamics too [26]. A detailed numerical analysis of 2-lump scattering within the geodesic approximation followed [7, as well as generalizations to the $\mathbb{C} P^{N}$ models 22 . One technical problem encountered in all these studies is that $\mathbb{C} P^{N}$ lumps have $L^{2}$-infinite zero modes on $\mathbb{C}$, so the $L^{2}$ metric is only well-defined on the leaves of a foliation of $\mathrm{M}_{n}$, rather than $\mathrm{M}_{n}$ itself. One interprets this physically as saying that certain parameters in the static $n$-lump solution are frozen to constant values by infinite inertia. For example, the width of a single $\mathbb{C} P^{1}$ lump is a free parameter, but is frozen in this fashion. Unfortunately, this freezing appears to be an artifact of the approximation numerical solutions suggest that a single lump may expand or contract according to the gen- 
uine field dynamics. So the geodesic approximation is rather pathological for these models. It is therefore interesting to consider situations where physical space is compact, since the $L^{2}$ metric is then guaranteed to be well-defined. In particular, $\mathbb{C} P^{1}$ lumps on $S^{2}$ and $T^{2}$ have been studied, and quite a lot is known about the corresponding $L^{2}$ geometries [19, 20, 21.

The question remains, of course, whether geodesic flow in $\mathrm{M}_{n}$ really does closely approximate low-energy $n$-lump dynamics in these theories. For 2-vortex and 2-monopole dynamics, rigorous results supporting the geodesic approximation have been proved by Stuart [23, 24]. He has shown that the solution with initial data $\phi(0) \in \mathrm{M}_{2}, \dot{\phi}(0) \in T_{\phi(0)} \mathrm{M}_{2}$ of order $\epsilon$ (where $\epsilon>0$ is small), stays pointwise close (order $\epsilon^{2}$ ) to its corresponding geodesic in $M_{2}$ for a time of order $\epsilon^{-1}$. The key idea in Stuart's analysis is to separate the dynamics into slow and fast time-varying modes by means of the following projection: the true solution $\phi(t)$ is projected onto $\mathrm{M}_{2}$ to obtain a slow trajectory $\widetilde{\phi}$

$$
\phi(t)=\widetilde{\phi}(\epsilon t)+\epsilon^{2} V(t)
$$

and a fast varying "error" $V(t)$, the projection being chosen so that $V(t)$ is always $L^{2}$ orthogonal to $T_{\widetilde{\phi}(\epsilon t)} \mathrm{M}_{2}$. Stuart goes on to prove that an appropriate Sobolev norm of $V(t)$ remains bounded for times of order $\epsilon^{-1}$ by bounding this norm in terms of Hess $\tilde{\phi}$, the quadratic form associated with the second variation of the potential energy functional of the model, which is slowly varying by virtue of energy conservation. It follows that, as expected, the true solution remains close to $\mathrm{M}_{2}$. That the projected trajectory $\widetilde{\phi}$ remains close to geodesic is proved as a separate step.

The whole analysis relies on one's ability to control Sobolev norms of the error in terms of the Hessian. More precisely, the following coercivity property of Hess is crucial:

There exists a constant $\tau>0$ such that for all $\widetilde{\phi} \in \mathrm{M}_{2}$ and all $V \in H^{1}$ with $V \perp_{L^{2}} T_{\tilde{\phi}} \mathrm{M}_{2}$, $\operatorname{Hess}_{\tilde{\phi}}(V, V) \geq \tau\|V\|_{H^{1}}^{2}$.

This turns out to be slightly easier to prove for vortices than monopoles since the $L^{2}$ spectrum of small oscillations about a static vortex has a mass gap due to the Higgs mechanism. No such gap occurs for monopoles.

How much of this framework carries over to sigma models? Since the target space has no linear structure, equation (1.1) makes no sense as it stands, and must be replaced. We suggest here that the correct replacement is

$$
\phi(t)=\exp _{\widetilde{\phi}(\epsilon t)} \epsilon^{2} V(t)
$$

where $\exp : T N \rightarrow N$ is the exponential map on the target space $N$. Once again, we should choose $V(t)$ always to be $L^{2}$ orthogonal to $T_{\widetilde{\phi}(\epsilon t)} \mathrm{M}_{n}$. In this context, we will prove an analogue of Stuart's coercivity lemma for any holomorphic map $\widetilde{\phi}$ between compact Kähler manifolds, namely, there exists $\tau(\widetilde{\phi})>0$ such that for all $V \in H^{1}, V \perp_{L^{2}} T_{\widetilde{\phi}} \mathrm{M}_{n}$,

$$
\operatorname{Hess}_{\tilde{\phi}}(V, V) \geq \tau(\widetilde{\phi})\|V\|_{H^{1}}^{2}
$$

The important difference from Stuart's result is that the constant $\tau$ depends on $\widetilde{\phi}$, that is, 
varies with position in $\mathrm{M}_{n}$. We may define the optimal constant

$$
\tau(\widetilde{\phi})=\inf \left\{\frac{\operatorname{Hess}_{\widetilde{\phi}}(V, V)}{\|V\|_{H^{1}}^{2}}: V \neq 0, V \perp_{L^{2}} T_{\widetilde{\phi}} \mathrm{M}_{n}\right\}>0 .
$$

We prove a result (Theorem [6) which gives sufficient conditions that $\tau$ depend continuously on $\widetilde{\phi}$, and verify that those conditions hold in the main examples of interest to us. It turns out that $\mathrm{M}_{n}$ for sigma models is generically non-compact, so continuity of $\tau(\widetilde{\phi})$ does not guarantee a global bound. In fact, we will show by means of an explicit counterexample, that $\tau(\widetilde{\phi})>0$ is not necessarily bounded away from zero. The counterexample occurs in the simplest nontrivial case, namely the one-lump moduli space of the $\mathbb{C} P^{1}$ model on $S^{2}$, but we believe it is indicative of a generic phenomenon for the $\underset{\sim}{\mathbb{C}} P^{N}$ models on any compact Riemann surface. Roughly speaking, $\tau(\widetilde{\phi}) \rightarrow 0$ as the lump $\widetilde{\phi}$ shrinks to zero size. One would expect this to happen quite generically in $\mathrm{M}_{n}$, wherever a family of holomorphic maps degenerates so that a single isolated lump collapses. This suggests we are never likely to have a global bounding constant $\tau$ on $\mathrm{M}_{n}$ as obtained by Stuart for vortices and monopoles. Consequently, the best result one could hope for from an analysis of this type for lumps is that the geodesic approximation is good for a time of order $T \epsilon^{-1}$, where $T$ is some increasing function of $\inf _{t} \tau(\widetilde{\phi}(\epsilon t))$. In this case, the approximation would work well (in the usual sense) so long as the projected geodesic stays away from $\partial_{\infty} \mathbf{M}_{n}$, the boundary at infinity of $\mathbf{M}_{n}$, where $\tau \rightarrow 0$. In particular it seems very unlikely that geodesic flow provides a good approximation to the process of single lump collapse itself. Two independent numerical studies of singularity formation in the $\mathbb{C} P^{1}$ model on the plane support this pessimistic assessment [3, 10].

If we impose extra symmetry on our system, in other words, restrict attention to an admissible equivariance class, then the projected map $\widetilde{\phi}$ is confined to a totally geodesic submanifold $\mathrm{M}_{n}^{e q}$ of $\mathrm{M}_{n}$, and the error $V$ is confined to an infinite dimensional subspace $H_{e q}^{1}$ of $H^{1}\left(\widetilde{\phi}^{*} T N\right)$. We may define an equivariant version $\tau^{e q}$ of $\tau$ by taking the infimum only over sections $V \in H_{e q}^{1}$. Clearly $\tau^{e q}(\widetilde{\phi}) \geq \tau(\widetilde{\phi})>0$. For a certain equivariance class for $n$-lump dynamics in the $\mathbb{C} P^{1}$ model on $S^{2}$, one can prove that $\tau^{e q}$ is continuous on $\mathrm{M}_{n}^{e q}$, which is again noncompact. We will present numerical evidence that $\tau^{e q}(\widetilde{\phi})$ is globally bounded away from zero for $n \geq 2$ in this class. The point is that only coincident $n$-lump collapse can occur within this particular equivariance class, and the problem of vanishing $\tau$ does not appear to happen for such collapse. It is possible, therefore, that the geodesic approximation does give a good model of equivariant multilump collapse, though one should be cautious: there is ample scope for other aspects of Stuart's method to break down as singularities form. There are some grounds for optimism. A recent careful numerical study of lump collapse by Linhart and Sadun found that single lump collapse on the plane differed significantly from that predicted by a truncated geodesic approximation, while coincident 2-lump collapse did not [10].

The rest of this paper is structured as follows. In section 2 we introduce the nonlinear sigma models of interest and briefly review some of their standard properties. We focus in particular on the $\mathbb{C} P^{1}$ model on $S^{2}$, describing what is known about the $L^{2}$ geometry on $\mathrm{M}_{n}$ in this case, especially for $n=1$. We present a new result on generic behaviour of the geodesic flow in $\mathrm{M}_{1}$ which implies that (within the geodesic approximation) a single lump generically does not travel along great circles on $S^{2}$. Precise restrictions on the set of non-generic initial data 
are given. In section 3 we formulate and prove the main result of the paper, that the Hessian for these models is coercive on $H^{1}$, in the sense described above. In section 4 we establish a simple sufficient condition for $\tau(\phi)$ to depend continuously on $\widetilde{\phi} \in \mathrm{M}_{n}$. In section 5 we show that this condition is met in the case of $\mathrm{M}_{1}$ for the $\mathbb{C} P^{1}$ model on $S^{2}$. We go on to prove that $\tau \rightarrow 0$ as $\widetilde{\phi}$ approaches $\partial_{\infty} \mathbf{M}_{1}$, so global coercivity fails in this case. Finally, in section [6] we consider the case of the $n$-lump sector of the $\mathbb{C} P^{1}$ model on $S^{2}$ within a given equivariance class, showing that $\tau^{e q}$ is continuous. We conjecture that Hess ${ }^{e q}$ is globally coercive for $n \geq 2$ and present some numerical results in support of this conjecture.

\section{The $\mathbb{C} P^{1}$ model on $S^{2}$}

For our purposes, a nonlinear sigma model consists of a single field $\phi: \mathbb{R} \times M \rightarrow N$, where $(M, g)$ and $(N, h)$ are compact Kähler manifolds, $M$ represents physical space, $\mathbb{R} \times M$ is spacetime, equipped with the Lorentzian pseudometric $\eta=d t^{2}-g$ and $N$ is the target space. Solutions of the model are local extremals of the action

$$
S[\phi]=\frac{1}{2} \int_{\mathbb{R} \times M} \sum_{\alpha} \frac{\left|d \phi E_{\alpha}\right|^{2}}{\left|E_{\alpha}\right|^{2}}=\int_{\mathbb{R}}\left(\frac{1}{2} \int_{M}|\dot{\phi}|^{2}-E[\phi]\right)
$$

where $E_{0}=\partial / \partial t, E_{1}, \cdots, E_{m}$ is an orthonormal basis of vector fields on $\mathbb{R} \times M$ and $E[\phi]$ is the harmonic map energy functional for maps $M \rightarrow N$. Such solutions are called wave maps in the geometric analysis literature, by analogy with harmonic maps [18. Indeed, static wave maps are precisely harmonic maps and hence have been the focus of intense study. In particular, Lichnerowicz showed that if a homotopy class $[\phi]$ contains \pm -holomorphic representatives, then these minimize energy within that class [9]. So the moduli space of interest within a given class is $\mathrm{M}_{[\phi]}=\mathrm{Hol}_{[\phi]}(M, N)$. For the sake of generality, we will state and prove the coercivity lemma for the Hessian in this general context.

It is important when using the geodesic approximation to know that $\mathrm{M}_{[\phi]}$ is a finite dimensional smooth manifold. This is not always true in the general case of holomorphic maps between Kähler manifolds. We discuss this question in more detail in section 3, briefly summarizing some results in the harmonic maps and algebraic geometry literature which allow us to identify classes of sigma model whose moduli spaces are smooth manifolds. For the moment we note that the particular case we have most directly in mind, namely $M=N=S^{2}$, the $\mathbb{C} P^{1}$ model on the two-sphere, certainly does have this property.

We now consider the case $M=N=S^{2}$ in more detail. Each homotopy class $[\phi] \in \pi_{2}\left(S^{2}\right)$ is labelled by the degree of $\phi$, an integer $n$, which without loss of generality we may assume is nonnegative. The degree $n$ is interpreted physically as the net lump number of the configuration $\phi$. The space $\mathrm{M}_{n}$ of degree $n$ holomorphic maps $S^{2} \rightarrow S^{2}$ is easily constructed explicitly. Choosing stereographic coordinates $z, W \in \mathbb{C}$ on both $M$ and $N$, such a map is rational of degree $n$,

$$
\phi: z \mapsto W=\frac{a_{1} z^{n}+\cdots+a_{n+1}}{a_{n+2} z^{n}+\cdots+a_{2 n+2}}
$$

where $a_{i}$ are $2 n+2$ complex constants, $a_{1}$ and $a_{n+2}$ do not both vanish, and the numerator and denominator have no common roots. Since $\left(a_{1}, \ldots, a_{2 n+2}\right)$ and $\left(\xi a_{1}, \ldots, \xi a_{2 n+2}\right)$ give the 
same map for all $\xi \in \mathbb{C} \backslash\{0\}$, we have a natural identification of $\mathrm{M}_{n}$ with a dense open subset of $\mathbb{C} P^{2 n+1}$, whence it inherits a natural topology and complex structure.

The metric of interest $\gamma$, does not descend from the inclusion $\mathrm{M}_{n} \subset \mathbb{C} P^{2 n+1}$ however. To define it, one must think of a tangent vector $X \in T_{\phi} \mathrm{M}_{n}$ as a zero mode of the Cauchy-Riemann equations for maps $M \rightarrow N$ at the map $\phi$. Such a zero mode is a smooth section of $\phi^{*} T N$, the pullback of the tangent bundle of $N$ by the map $\phi$, that is, a rule which assigns to each $p \in M$ a vector $V(p) \in T_{\phi(p)} N$. We may define the $L^{2}$ inner product between any pair of sufficiently regular sections $X, Y$ of $\phi^{*} T N$ by taking their fibrewise inner product in $T_{\phi(p)} N$, then integrating over $M$,

$$
\langle X, Y\rangle_{L^{2}}=\int_{M} h(X, Y)
$$

The $L^{2}$ metric on $M_{n}$ is simply the restriction of $\langle\cdot, \cdot\rangle_{L^{2}}$ to zero modes. In more concrete terms, one can in principle compute explicit formulae for $\gamma$ by choosing local coordinates $q^{i}$ on $\mathrm{M}_{n}$ (for example, the real and imaginary parts of $a_{i} / a_{1}$, on the chart where $a_{1} \neq 0$ ) and expressing the map $\phi\left(\left\{q^{i}\right\}\right)$ as an explicit function $W\left(z,\left\{q^{i}\right\}\right)$, so that

$$
\gamma=\sum_{i j} \gamma_{i j} d q^{i} d q^{j}, \quad \gamma_{i j}=\int_{\mathbb{C}} \frac{d z d \bar{z}}{\left(1+|z|^{2}\right)^{2}} \frac{1}{\left(1+|W|^{2}\right)^{2}} \frac{\partial W}{\partial q^{i}} \frac{\overline{\partial W}}{\partial q^{j}}
$$

In practice, of course, the integrals involved are almost always intractable.

Certainly, the $L^{2}$ metric is a natural way of geometrizing $\mathrm{M}_{n}$. More importantly, it is the Riemannian metric descending from the restriction of the kinetic energy functional $\frac{1}{2} \int_{M}|\dot{\phi}|^{2}$ to $\mathrm{M}_{n}$, and hence the metric whose geodesics are thought to model slow lump dynamics. We shall briefly review what is known about the Riemannian manifold $\left(\mathrm{M}_{n}, \gamma\right)$, and prove a new result about the generic behaviour of geodesics in $\left(\mathrm{M}_{1}, \gamma\right)$.

First, $\left(\mathrm{M}_{n}, \gamma\right)$ is manifestly Hermitian, and is in fact Kähler. This was long suspected, owing to a rather general formal argument of Ruback [15], and has recently been proved rigorously 21]. It is also known that $\left(\mathrm{M}_{n}, \gamma\right)$ is geodesically incomplete [16]. For odd $n,\left(\mathrm{M}_{n}, \gamma\right)$ contains a totally geodesic Lagrangian submanifold naturally identified with the moduli space of static $\mathbb{R} P^{2} n$-lumps on $\mathbb{R} P^{2}$; for $n \geq 3$ this submanifold is also incomplete.

There is an isometric action of $G=S O(3) \times S O(3)$ on $\mathrm{M}_{n}$, induced by the natural $S O(3)$ actions on the domain and target spheres, which on $\mathrm{M}_{1}$ has cohomogeneity 1 (generic $G$ orbits have codimension 1), and in fact, almost completely determines $\gamma$. Consequently, an explicit formula for $\gamma$ is known in this case, and the geometry is particularly well understood. For $n=1$, the no common roots condition on the rational map $W(z)=\left(a_{1} z+a_{2}\right) /\left(a_{3} z+a_{4}\right)$ is $a_{1} a_{4}-a_{2} a_{3} \neq 0$, so we may identify each map with a projective equivalence class $[L]$ of $G L(2, \mathbb{C})$ matrices. Hence $\mathrm{M}_{1} \cong P L(2, \mathbb{C})$. By identifying $S^{2}$ with the unit sphere in $\mathbb{R}^{3} \cong \mathfrak{s u}(2)$ in the usual way, we may identify the $S O(3)$ action on $S^{2}$ with the adjoint $S U(2)$ action, so that $\gamma$ regarded as a metric on $P L(2, \mathbb{C})$ is invariant under the left and right $P U(2)$ actions:

$$
\left(\left[U_{1}\right],\left[U_{2}\right]\right):[L] \mapsto\left[U_{1} L U_{2}\right] .
$$

Now every $[L] \in P L(2, \mathbb{C})$ has a unique polar decomposition

$$
[L]=\left[U\left(\sqrt{1+\lambda^{2}} \mathbb{I}_{2}+\boldsymbol{\lambda} \cdot \boldsymbol{\tau}\right)\right]
$$


where $([U], \boldsymbol{\lambda}) \in P U(2) \times \mathbb{R}^{3}, \lambda=|\boldsymbol{\lambda}|$, and $\tau_{1}, \tau_{2}, \tau_{3}$ are the Pauli spin matrices. Hence $\mathrm{M}_{1} \cong P U(2) \times \mathbb{R}^{3} \cong S O(3) \times \mathbb{R}^{3}$. Physically, the lump corresponding to $([U], \boldsymbol{\lambda})$ has maximum energy density at $-\boldsymbol{\lambda} / \lambda \in S^{2}$, sharpness proportional to $\lambda$ and internal orientation $[U]$. The $\lambda=0$ lumps have uniform energy density. The $G$ action in this coordinate system is

$$
\left(\left[U_{1}\right],\left[U_{2}\right]\right):([U], \boldsymbol{\lambda}) \mapsto\left(\left[U_{1} U U_{2}\right], A d_{U_{2}} \boldsymbol{\lambda}\right)
$$

where again we have used $\mathbb{R}^{3} \cong \mathfrak{s u}(2)$ to identify the fundamental $S O(3)$ action on $\mathbb{R}^{3}$ with the adjoint $S U(2)$ action on $\mathfrak{s u}(2)$. From this we see that the $G$-orbits are level sets of $\lambda$, generically diffeomorphic to $S O(3) \times S^{2}$ (when $\lambda>0$ ), the only exception being $\lambda=0$, which is diffeomorphic to $S O(3)$.

In [21] it was proved that every $G$ invariant Kähler metric on $\mathrm{M}_{1}$ may be written

$$
\gamma=A_{1} d \boldsymbol{\lambda} \cdot d \boldsymbol{\lambda}+A_{2}(\boldsymbol{\lambda} \cdot d \boldsymbol{\lambda})^{2}+A_{3} \boldsymbol{\sigma} \cdot \boldsymbol{\sigma}+A_{4}(\boldsymbol{\lambda} \cdot \boldsymbol{\sigma})^{2}+A_{1} \boldsymbol{\lambda} \cdot(\boldsymbol{\sigma} \times d \boldsymbol{\lambda})
$$

where $A_{1}, \ldots, A_{4}$ are smooth functions of $\lambda$ only, all determined from the single function $A_{1}=A(\lambda)$ by the relations

$$
A_{2}=\frac{A(\lambda)}{1+\lambda^{2}}+\frac{A^{\prime}(\lambda)}{\lambda}, \quad A_{3}=\frac{1}{4}\left(1+2 \lambda^{2}\right) A(\lambda), \quad A_{4}=\frac{1}{4 \lambda}\left(1+\lambda^{2}\right) A^{\prime}(\lambda) .
$$

Here $\sigma_{1}, \sigma_{2}, \sigma_{3}$ are the left invariant one forms on $S O(3)$ dual to the basis $\left\{\frac{i}{2} \tau_{a}: a=1,2,3\right\}$ for $\mathfrak{s u}(2) \cong \mathfrak{s o}(3)$. For the $L^{2}$ metric, one finds that

$$
A=\frac{4 \pi \mu\left[\mu^{4}-4 \mu^{2} \log \mu-1\right]}{\left(\mu^{2}-1\right)^{3}}, \quad \mu=\left(\sqrt{1+\lambda^{2}}+\lambda\right)^{2} .
$$

It follows from these formulae that $\left(\mathrm{M}_{1}, \gamma\right)$ has finite volume and diameter, is Ricci positive and has unbounded scalar and holomorphic sectional curvatures. Examining the large $\lambda$ behaviour of $\gamma$, one finds that $\partial_{\infty} \mathrm{M}_{1}$, the boundary at infinity of $\mathrm{M}_{1}$ is $S^{2} \times S^{2}$. This is natural in two ways: a point in $\partial_{\infty} \mathrm{M}_{1}$ should be thought of as a collapsed lump whose width has shrunk to zero. Such a lump is specified by a pair of points $p, p^{\prime}$ in $S^{2}$ because every point except one, $p$, in the domain gets mapped to the same point $p^{\prime}$ in the codomain, while $p$ gets mapped to the antipodal point $-p^{\prime}$. Second, the complex codimension 1 algebraic variety $a_{1} a_{4}-a_{2} a_{3}=0$ complementary to $\mathrm{M}_{1}$ in $\mathbb{C} P^{3}$ is biholomorphic to $\mathbb{C} P^{1} \times \mathbb{C} P^{1}$, being the image of the holomorphic embedding

$$
\left(\left[x_{1}, x_{2}\right],\left[y_{1}, y_{2}\right]\right) \mapsto\left[x_{1} y_{2}, x_{1} y_{1}, x_{2} y_{2}, x_{2} y_{1}\right]
$$

Since $\operatorname{diam}\left(\mathrm{M}_{1}, \gamma\right)<\infty, \partial_{\infty} \mathrm{M}_{1}$ lies at finite proper distance, so geodesics may reach it in finite time, the origin of the incompleteness already noted. Given that $\partial_{\infty} \mathrm{M}_{1}$ has (real) codimension 2 , however, one would expect geodesics to miss $\partial_{\infty} \mathrm{M}_{1}$ generically. More precisely, one would expect the subset of $T \mathrm{M}_{1}$ consisting of initial data of geodesics which do escape to infinity to have zero measure with respect to the natural measure inherited from $\gamma$.

Geodesic flow in $\left(M_{1}, \gamma\right)$ was studied in detail in [19]. It turns out to be surprisingly complicated given the homogeneity and isotropy of the domain of the sigma model, $S^{2}$. Geodesics were found for which the lump spins internally and oscillates between antipodal points. Other 
geodesics were found where the lump travels along a great circle in $S^{2}$, its speed and shape undergoing complicated periodic oscillations. Geodesics do exist for which the lump simply traverses a great circle at constant speed and shape, but the initial data to generate such motion must be chosen very carefully. Nevertheless, all the geodesics found in [19] confined the lump's position $-\boldsymbol{\lambda} / \boldsymbol{\lambda}$ to some great circle for all time. More precisely, they all confined $\boldsymbol{\lambda}(t)$ to some plane through the origin. The geodesics themselves were obtained by reducing the geodesic problem to low dimensional totally geodesic submanifolds, the fixed point sets of discrete isometries. The question arises, then, whether this "planar property" of the geodesic flow is an artifact of the exceptional symmetries enjoyed by these geodesics, or is a general feature of the dynamics. We shall now prove that the former is the case.

By the $G$ invariance of the metric, it suffices to determine which initial velocities $u \in T_{x(0)} H$ at the point $x(0)=\left(\left[\mathbb{I}_{2}\right],(0,0, \lambda)\right.$ tangent to the hypersurface $H=\left\{([U], \boldsymbol{\lambda}): \lambda_{2}=0\right\}$ generate geodesics which remain in $H$. We shall call such an initial velocity $u$ a "good" vector. Clearly the set of good initial velocities is conical by time-scaling invariance of geodesic flow. By this, we mean that if $u \in T_{x(0)} H$ is good, so is every vector $\xi u \in T_{x(0)} H, \xi \in[0, \infty)$, on the ray containing $u$. The question is then whether the link of this cone of good velocity vectors, that is, its intersection with the unit 4-sphere in $T_{x(0)} H$, has non-vanishing measure in $S^{4}$.

Theorem 1 Let $u \in T_{x(0)} H$ generate a geodesic through $x(0)$ which remains in $H$. Then $u$ lies in a codimension 1 cone in $T_{x(0)} H$. For generic $x(0) \in H$, the link of this cone is the suspension of a two-torus. There is at most a nowhere dense subset of $H$ on which the link of the cone is $S^{3} \cup S^{3}$, two three-spheres intersecting in an equatorial two-sphere.

Proof: Let $N$ be a nonvanishing (but not necessarily unit) normal on $H$. Then if $x(t)$ remains in $H, \gamma(\dot{x}(t), N(x(t)))=0$ for all $t$. Differentiating this at $t=0$ and using the fact that $\dot{x}(t)$ is parallel for a geodesic, one finds that $\gamma\left(\dot{x}(0), \nabla_{\dot{x}(0)} N\right)=0$, where $\nabla$ is the Levi-Civita connexion. Hence, $u=\dot{x}(0)$ must lie in the null space of the symmetric bilinear form

$$
B(u, v)=\gamma\left(u, \nabla_{v} N\right)
$$

that is $B(u, u)=0$. Clearly this null space is conical. We seek to understand the link of this cone. In this case $\left(H=\left\{\lambda_{2}=0\right\}\right)$, we may choose

$$
N=A_{3} \frac{\partial}{\partial \lambda_{2}}+\frac{1}{2} A_{1}\left(-\lambda_{3} \theta_{1}+\lambda_{1} \theta_{3}\right)
$$

as our normal field, where $\theta_{a}$ are the left-invariant vector fields on $S O(3)$ dual to $\sigma_{a}$. We may compute $B(u, v)$ by extending $u, v$ to vector fields $U, V$ on $\mathrm{M}_{1}$, then using the usual formula for $\nabla$ and symmetry of $B$ [27], to yield

$$
B(u, v)=\left.\frac{1}{2} N[\gamma(U, V)]\right|_{x(0)}+\frac{1}{2} \gamma\left(\left.[U, N]\right|_{x(0)}, v\right)+\frac{1}{2} \gamma\left(\left.[V, N]\right|_{x(0)}, u\right) .
$$

A straightforward but lengthy calculation then shows that

$$
B=f_{1}(\lambda) d \lambda_{1} \sigma_{3}+f_{2}(\lambda) d \lambda_{3} \sigma_{1}+f_{3}(\lambda) \sigma_{2} \sigma_{3}
$$


where

$$
f_{1}=\frac{1}{8}\left(1+\lambda^{2}\right)\left(2 A+\lambda A^{\prime}\right), \quad f_{2}=-\frac{1}{4}\left(1+2 \lambda^{2}\right) A\left(A+\lambda A^{\prime}\right), \quad f_{3}=\frac{1}{16}\left(1+\lambda^{2}\right) A A^{\prime},
$$

at the specific point $x(0)=\left(\left[\mathbb{I}_{2}\right],(0,0, \lambda)\right)$. By computing eigenvalues, one sees that, with respect to some orthonormal basis for $T_{x(0)} H$,

$$
B(u, u)=f_{2}(\lambda)\left(u_{2}^{2}-u_{3}^{2}\right)+\sqrt{f_{1}(\lambda)^{2}+f_{3}(\lambda)^{2}}\left(u_{4}^{2}-u_{5}^{2}\right) .
$$

Note that

$$
\frac{8 f_{1}(\lambda)}{1+\lambda^{2}}=2 A+\lambda A^{\prime}>\frac{2 \lambda^{1}+1}{\lambda^{2}+1} A+\lambda A^{\prime}=\gamma\left(\frac{\partial}{\partial \lambda_{3}}, \frac{\partial}{\partial \lambda_{3}}\right)>0
$$

so $f_{1}(\lambda)$ never vanishes. Consider the set $L=f_{2}^{-1}(0) \subset[0, \infty)$. If $L$ were dense at $\lambda_{0}$, then by continuity of $A+\lambda A^{\prime}$, there would be an open interval containing $\lambda_{0}$ such that $A=$ $-\log \lambda+$ constant. Given the formula (2.10) $A$ clearly does not coincide with $-\log \lambda+$ constant on any interval, so no such $\lambda_{0}$ exists. Hence the set $L$ is nowhere dense.

Let $\lambda \notin L$. By means of a $\lambda$ dependent rescaling of the basis, we see that the null space is the locus of the equation

$$
\tilde{u}_{2}^{2}+\tilde{u}_{4}^{2}=\tilde{u}_{3}^{2}+\tilde{u}_{5}^{2} .
$$

Clearly $\tilde{u}_{1}$ may take any value, while $\left(\tilde{u}_{2}, \tilde{u}_{3}, \tilde{u}_{4}, \tilde{u}_{5}\right)$ lies on a cone in $\mathbb{R}^{4}$ whose link is a twotorus. Alternatively, we may think of the unit sphere in $\mathbb{R}^{5}$ as the suspension of the unit sphere in $\mathbb{R}^{4}$ along the $\tilde{u}_{1}$ direction. Then the intersection of the link of the null space with each 3 sphere of constant $\tilde{u}_{1} \in[-1,1]$ is a scaled two-torus. Assembling the slices together we see that the whole null space has a link which is topologically the suspension of a two-torus [6]. Either way of viewing the null set, it is clearly a codimension 1 cone in $T_{x(0)} H$ as claimed.

It remains to consider the non-generic case, $\lambda \in L$, for which $f_{2}(\lambda)=0$. In this case, since $f_{1}(\lambda) \neq 0$, the cone consists of all vectors for which $u_{4}= \pm u_{5}$. The link is the intersection of $S^{4}$ with the union of the two orthogonal hyperplanes $\left(u_{4} \pm u_{5}\right)=0$, which is manifestly a union of two three-spheres intersecting in an equatorial two sphere $\left(u_{4}=u_{5}=0\right)$.

We should point out that $u \in \operatorname{null} B$ is a necessary but not sufficient condition for the geodesic with initial velocity $u$ to stay in $H$. It is not certain that the cone of good velocities is all of null $B$, therefore. Given the explicit formula for $A$, one would expect to be able to improve the characterization of the non-generic subset of $H$ (on which null $B$ has link $S^{3} \cup S^{3}$ ) from nowhere dense to discrete or finite. Indeed, one may check graphically that $f_{2}(\lambda)=0$ has only one solution ( $\lambda=0.881$ to 3 decimal places), so the nongeneric set consists of a single $G$ orbit, $S^{1} \times S O(3)$, in $H$.

Corollary 2 Generically geodesics in $\left(\mathrm{M}_{1}, \gamma\right)$ do not confine $\boldsymbol{\lambda}(t)$ to a plane through $\mathbf{0}$. Consequently, single lumps generically do not stay on great circles while moving on $S^{2}$.

Of course, this corollary refers to the lump dynamics within the geodesic approximation. The question remains: does this give a good model of the full field dynamics? 


\section{Coercivity of the Hessian}

We wish to consider the wave map problem for maps $\left(\mathbb{R} \times M, d t^{2}-g\right) \rightarrow(N, h)$, where $(M, g),(N, h)$ are compact Kähler, and the initial data have a certain Sobolev regularity and are close to holomorphic, in a sense to be made precise. The eventual aim is to prove that such wave maps stay close to $\mathrm{Hol}_{[\phi]}(M, N)$ and that their closest trajectory in $\mathrm{Hol}_{[\phi]}(M, N)$ is close to a geodesic. In this section we will prove the main analytic ingredient needed for such a programme, along the lines of Stuart's work on vortices.

The first thing to note is that the Cauchy problem for such wave maps with initial data $\left(\phi_{0}, \dot{\phi}_{0}\right) \in H^{k} \oplus H^{k-1}$ on the time slice $\{0\} \times M$ is well posed, that is, has a unique solution in the same Sobolev space, with $t \mapsto(\phi(t), \dot{\phi}(t))$ continuous, at least on some open time interval, $t \in(-\epsilon, \epsilon)$, by work of Choquet-Bruhat [4]. Here $H^{k}$ denotes the space of maps $M \rightarrow \mathbb{R}^{p}$ which are $H^{k}$ in the usual sense $\left(\phi, \nabla \phi, \ldots, \nabla^{k} \phi\right.$ are all $\left.L^{2}\right)$ and which take values on $N \subset \mathbb{R}^{p}$ (isometrically embedded in $\mathbb{R}^{p}$ ) almost everywhere. For our purposes, it is convenient to use an alternative intrinsic definition of $H^{k}(M, N)$, which is only well-defined for $k>\frac{1}{2} \operatorname{dim} M$. With this restriction on $k, H^{k}(M, N)$ naturally has the structure of a Hilbert manifold. Below we give a brief treatment of both the intrinsic definition and the Hilbert manifold structure of $H^{k}(M, N)$. For a complete treatment we refer the reader to Palais [14.

A map $\phi: M \rightarrow N$ is said to belong to $H^{k}(M, N)$ if for any $p \in M$ and any chart $(U, \Phi)$ containing $p$, and any chart $(V, \Psi)$ containing $\phi(p)$, the map $\Psi \circ \phi \circ \Phi^{-1}: \Phi(U) \rightarrow \mathbb{R}^{n}$ belongs to $H^{k}\left(\Phi(U), \mathbb{R}^{n}\right)$. For this notion to be well-defined we need to ensure that composition by $C^{\infty}$ diffeomorphisms on the left and on the right takes an $H^{k}$ map to an $H^{k}$ map. For composition on the right this is true without any restriction on $k$. However, for left composition the same result only holds if we assume $k>\frac{1}{2} \operatorname{dim} M$ (for a discussion of both points see B.1.7 and B.1.9, p182 [13]). From now on we make a standing assumption that $k>\frac{1}{2} \operatorname{dim} M$, so that the intrinsic definition of $H^{k}(M, N)$ given above makes sense.

We now exhibit the structure of an infinite dimensional Hilbert manifold on $H^{k}(M, N)$. Standard facts on embeddings of Sobolev spaces and the density of smooth maps in Sobolev spaces prove the following: There are continuous inclusions $C^{\infty}(M, N) \hookrightarrow H^{k}(M, N) \hookrightarrow$ $C^{0}(M, N)$ and the first inclusion has dense image. From the latter fact it follows that it is sufficient to exhibit charts for $H^{k}(M, N)$ around only the $C^{\infty}$ maps.

Given $\phi \in C^{\infty}(M, N)$ the pullback bundle $\phi^{*} T N$ (i.e. the vector bundle over $M$ whose fibre at $m$ is the vector space $\left.T_{\phi(m)} N\right)$ comes equipped with a natural inner product $h \circ(\phi \times \phi)$ and compatible connexion $\nabla^{\phi}$, the pullback of the Levi-Civita connexion on $T N$. For any vector bundle $E$ over compact $M$ equipped with an inner product $<,>$ and compatible connection $\nabla$ there is a natural $H^{k}$ inner product on smooth sections of $E$ :

$$
<V, W>_{H^{k}}=\int_{M}<V, W>+\int_{M}<\nabla V, \nabla W>+\cdots+\int_{M}<\nabla^{k} V, \nabla^{k} W>.
$$

$H^{k}(E)$ is then defined as the set of finite $H^{k}$ norm elements of the completion of $C^{\infty}(E)$ with respect to the norm $\|.\|_{H^{k}}$. For $M$ compact, this definition of $H^{k}(E)$ is equivalent to the following alternative definition: given any choice of local coordinates on $M$ and associated bundle trivializations for $E$, a section belongs to $H^{k}(E)$ if it is represented by locally $H^{k}$ functions in these trivializations. The difference is that the connexion-dependent definition 
gives a preferred inner product on $H^{k}(E)$, i.e. $\left(H^{k}(E),\|\cdot\|_{H^{k}}\right)$ is naturally a Hilbert space.

The point is that $H^{k}\left(\phi^{*} T N\right)$ is the local model space for the Hilbert manifold $H^{k}(M, N)$. For each $\phi \in C^{\infty}(M, N)$, there is a map $\exp _{\phi}: H^{k}\left(\phi^{*} T N\right) \rightarrow H^{k}(M, N)$ given by $V(p) \mapsto$ $\exp _{\phi(p)} V(p)$, where exp :TN $\rightarrow N$ is the exponential map on $(N, h)$. It can be shown that $\exp _{\phi}$ maps a neighbourhood of 0 in the Hilbert space $H^{k}\left(\phi^{*} T N\right)$ bijectively to a neighbourhood of $\phi$ in $H^{k}(M, N)$. Hence for each $\phi \in C^{\infty}(M, N)$ there exists some $\epsilon>0$, so that one can define a chart $\left(U_{\phi}, \exp _{\phi}^{-1}\right)$ based at $\phi$ where

$$
U_{\phi}=\left\{\exp _{\phi}(V): V \in H^{k}\left(\phi^{*} T N\right),\|V\|_{H^{k}}<\epsilon\right\} .
$$

The local homeomorphism $U_{\phi} \rightarrow B_{\epsilon}(0) \subset H^{k}\left(\phi^{*} T N\right)$ is simply given by $\exp _{\phi}^{-1}$. So we identify $H^{k}$ maps close to $\phi$ with $H^{k}$ sections of $\phi^{*} T N$ by deforming $\phi$ : the deformed map $\exp _{\phi}(V)$ maps each $p \in M$ to the point in $N$ reached by travelling for unit time along the geodesic with initial data $(\phi(p), V(p))$.

It can be shown that for any two maps $\phi_{1}, \phi_{2} \in C^{\infty}(M, N)$ the change of charts map $\exp _{\phi_{2}}^{-1} \circ \exp _{\phi_{1}}: \exp _{\phi_{1}}^{-1}\left(U_{\phi_{1}} \cap U_{\phi_{2}}\right) \rightarrow \exp _{\phi_{2}}^{-1}\left(U_{\phi_{1}} \cap U_{\phi_{2}}\right)$ is a diffeomorphism between open sets in the Hilbert spaces $H^{k}\left(\phi_{1}^{*} T N\right)$ and $H^{k}\left(\phi_{2}^{*} T N\right)$. It follows that the collection of charts $\left\{\left(U_{\phi}, \exp _{\phi}^{-1}\right) \mid \phi \in C^{\infty}(M, N)\right\}$ defines a differentiable structure on $H^{k}(M, N)$ with local model a Hilbert space, i.e. $H^{k}(M, N)$ is a Hilbert manifold. In fact, the differentiable structure can be shown to be independent of the metric $h$ on $N$ used to define $\exp _{\phi}$.

Given

- a holomorphic map $\widetilde{\phi}_{0} \in \operatorname{Hol}(M, N)$, and

- sections $V_{0}, X_{0}, Y_{0} \in H^{k}\left(\widetilde{\phi}_{0}^{*} T N\right)$, such that

- $V_{0}, Y_{0}$ are $L^{2}$ orthogonal to $T_{\widetilde{\phi}_{0}} \operatorname{Hol}(M, N)$, and

- $X_{0} \in T_{\widetilde{\phi}_{0}} \operatorname{Hol}(M, N)$

and $\epsilon>0$ small, the initial value problem with initial data

$$
\phi_{0}=\exp _{\widetilde{\phi}_{0}} \epsilon^{2} V_{0}, \quad \dot{\phi}_{0}=\epsilon X_{0}+\epsilon^{3} Y_{0}
$$

has a unique solution in $H^{k}$. The idea is to decompose this solution into $\widetilde{\phi}(t) \in \operatorname{Hol}(M, N)$ and $V(t) \in H^{k}\left(\widetilde{\phi}(t)^{*} T N\right) \cap T_{\widetilde{\phi}(t)} \operatorname{Hol}(M, N)^{\perp} L^{2}$ by

$$
\phi(t)=\exp _{\widetilde{\phi}(t)} \epsilon^{2} V(t)
$$

and then show that $\|V\|_{H^{k}}$ remains bounded for times of order $\epsilon^{-1}$. The starting point is to show that $\|V\|_{H^{1}}$ is controlled by $\operatorname{Hess}_{\tilde{\phi}}(V, V)$. From now on, all quantities will be considered at a fixed time, and we will denote the holomorphic base map of our local chart in $H^{k}$ by $\phi$ rather than $\widetilde{\phi}$, to simplify notation. 
Hess $_{\phi}(X, Y)$ is the second variation of the harmonic map energy $E$ at the holomorphic (hence harmonic) map $\phi$. Precisely, given a two-parameter variation $\phi_{s, t}$ of the map $\phi$ through smooth maps, with $d \phi_{0,0} \partial / \partial s=X, d \phi_{0,0} \partial / \partial t=Y \in \Gamma\left(\phi^{*} T N\right)$,

$$
\operatorname{Hess}_{\phi}(X, Y)=\left.\frac{\partial^{2} E\left[\phi_{s, t}\right]}{\partial s \partial t}\right|_{s=t=0} .
$$

There are two useful explicit formulae for Hess 25. The first uses only compactness of $M$, not the Kähler property. To write it down we must introduce two new objects. Let $E_{i}, i=1, \ldots, m$ be a local frame of smooth orthonormal vector fields on $M$. Then the rough Laplacian on sections of $\phi^{*} T N$ is the second order linear elliptic differential operator

$$
\Delta^{\phi} V=-\operatorname{tr}\left(\nabla^{\phi} \nabla^{\phi} V\right)=-\sum_{i=1}^{m}\left(\nabla^{\phi} \nabla^{\phi} V\right)\left(E_{i}, E_{i}\right) .
$$

Like the usual Laplacian (on functions or forms) $\Delta^{\phi}$ is a positive self-adjoint operator. Positivity follows from the identity

$$
\int_{M} h\left(V, \Delta^{\phi} V\right)=\frac{1}{2} \int_{M} \sum_{i} h\left(\nabla_{E_{i}}^{\phi} V, \nabla_{E_{i}}^{\phi} V\right) .
$$

We may define a (fibrewise linear) bundle map $\mathfrak{R}^{\phi}$ on $\phi^{*} T N$ by

$$
\mathfrak{R}^{\phi} V=\sum_{i=1}^{m} R^{N}\left(V, d \phi E_{i}\right) d \phi E_{i}
$$

where $R^{N}$ is the curvature tensor on $N$. Given these, the Hessian is

$$
\operatorname{Hess}_{\phi}(X, Y)=\int_{M} h\left(X, \mathfrak{J}^{\phi} Y\right)=\left\langle X, \mathfrak{J}^{\phi}\right\rangle_{L^{2}}, \quad \mathfrak{J}^{\phi}=\Delta^{\phi}-\mathfrak{R}^{\phi}
$$

The operator $\mathfrak{J}^{\phi}$, called the Jacobi operator, is itself second order, linear, elliptic and selfadjoint. It follows immediately from this formula that every harmonic map into a manifold of nonpositive sectional curvature is weakly stable (meaning $\operatorname{Hess}_{\phi}(V, V) \geq 0$ ).

In the case of interest to us, namely $M, N$ compact Kähler, and $\phi$ holomorphic, one can obtain a more useful and rather simpler formula for Hess. First one defines the Urakawa connexion on $\phi^{*} T N$,

$$
\left(\mathfrak{D}^{\phi} V\right)(X):=\nabla_{J^{M} X}^{\phi} V-J^{N} \nabla_{X}^{\phi} V
$$

$J^{M}, J^{N}$ being the almost complex structures on $M$ and $N$ [25]. Then

$$
\operatorname{Hess}_{\phi}(V, V)=\frac{1}{2}\left\|\mathfrak{D}^{\phi} V\right\|_{L^{2}}^{2}
$$

Weak stability of holomorphic maps follows immediately from (3.10).

$\mathfrak{D}^{\phi} V=0$ should be thought of as the linearized Bogomol'nyi equation, so every $V \in$ $\operatorname{ker} \mathfrak{D}^{\phi}=\operatorname{ker} \mathfrak{J}^{\phi}$ is a zero mode of the holomorphic map $\phi$. For the geodesic approximation 
to make sense, the space of holomorphic maps close to $\phi$ must be a smooth manifold whose tangent space at $\phi$ equals ker $\mathfrak{J}^{\phi}$. Sections of $\phi^{*} T N$ in the kernel of $\mathfrak{J}^{\phi}$ are called Jacobi fields along $\phi$. A Jacobi field is said to be integrable if it may be generated by a variation of $\phi$ through harmonic maps, and the map itself is said to be Jacobi integrable if all its Jacobi fields have this property. A fundamental theorem of Adams and Simon [1] states that a harmonic map $\phi$ between real analytic manifolds is Jacobi integrable if and only if the space of harmonic maps $C^{2, \alpha}$ close to $\phi$ is a smooth manifold with tangent space ker $\mathfrak{J}^{\phi}$ at $\phi$. Similar results hold in suitable Sobolev spaces of maps also. In the case where $M, N$ are Kähler (hence real analytic), Lichnerowicz showed that all harmonic deformations of a holomorphic map $\phi$ are holomorphic [9, so the space of interest to us, the space of holomorphic maps close to $\phi$, is a smooth manifold with tangent space ker $\mathfrak{J}^{\phi}$ at $\phi$, if and only if $\phi$ is Jacobi integrable. Jacobi integrability of harmonic maps is an active field of research whose current state is summarized in [8]. Particularly relevant to the present paper is a theorem of Wood and Lemaire which states that every holomorphic map $S^{2} \rightarrow \mathbb{C} P^{N}$ is Jacobi integrable. So the moduli space of holomorphic maps for the $\mathbb{C} P^{N}$ model on $S^{2}$ is smooth with tangent space ker $\mathfrak{J}^{\phi}$, as we require. A similar result holds for degree $n$ holomorphic maps $\Sigma \rightarrow \mathbb{C} P^{1}$, $\Sigma$ being a compact Riemann surface of genus $g$, provided $n>2 g-2$, by a standard application of the RiemannRoch theorem. So the $n$-lump moduli space of the $\mathbb{C} P^{1}$ model on $\Sigma$ also has the required property, with some low degree exceptions.

We may now state and prove our main result.

Theorem 3 (Coercivity of the Hessian) Let $\phi: M \rightarrow N$ be a holomorphic map between compact Kähler manifolds and Hess $_{\phi}$ be the Hessian of the harmonic map energy functional at $\phi$. Then there exists a constant $\tau(\phi)>0$ such that for all $V \in H^{1}\left(\phi^{*} T N\right)$ with $\left\langle V \text {, } \operatorname{ker} \mathfrak{D}^{\phi}\right\rangle_{L^{2}}=$ 0 ,

$$
\operatorname{Hess}_{\phi}(V, V) \geq \tau(\phi)\|V\|_{H^{1}}^{2}
$$

Proof: Both $H^{1}$ and $L^{2}$ are Hilbert spaces. We will use $\rightarrow$ and $\rightarrow$ to denote strong and weak convergence respectively, the space concerned being explicitly specified. Define the subset $S=\left\{V \in H^{1}:\|V\|_{H^{1}}^{2}=1, V \perp_{L^{2}} \operatorname{ker} \mathfrak{D}^{\phi}\right\}$ and the quantity

$$
\tau(\phi)=\inf _{V \in S} \operatorname{Hess}_{\phi}(V, V)=\inf _{V \in S} \frac{1}{2}\left\|\mathfrak{D}^{\phi} V\right\|_{L^{2}}^{2} \geq 0 .
$$

We claim that $\tau(\phi) \neq 0$. Assume this is false. Then there exists a sequence $V_{i} \in S$ such that $\mathfrak{D}^{\phi} V_{i} \stackrel{L^{2}}{\rightarrow} 0$. We will repeatedly extract (nested) subsequences from $V_{i}$, which we will always denote by the same symbol, $V_{i}$. Now $V_{i}$ is bounded in $H^{1}$ so by the Alaoglu theorem, there exists a subsequence $V_{i} \stackrel{H^{1}}{\rightarrow} V$, to some weak limit $V$. Since $\mathfrak{D}^{\phi}: H^{1} \rightarrow L^{2}$ is a bounded linear map, it is continuous with respect to the weak (and strong) topologies on $H^{1}, L^{2}$. It follows that $\mathfrak{D}^{\phi} V_{i} \stackrel{L^{2}}{\longrightarrow} \mathfrak{D}^{\phi} V$. But $\mathfrak{D}^{\phi} V_{i} \stackrel{L^{2}}{\rightarrow} 0 \Rightarrow \mathfrak{D}^{\phi} V_{i} \stackrel{L^{2}}{\rightarrow} 0 \Rightarrow \mathfrak{D}^{\phi} V=0$ by uniqueness of weak limits. Hence $V_{i} \stackrel{H^{1}}{\rightarrow} V \in \operatorname{ker} \mathfrak{D}^{\phi}$.

Now the inclusion $\iota: H^{1} \hookrightarrow L^{2}$ is compact by Rellich's lemma, so the bounded set $\left\{V_{i}\right\} \subset$ $H^{1}$ is compact in $L^{2}$. Hence, any sequence in $\left\{V_{i}\right\}$, for example $V_{i}$ itself, has a subsequence which is strongly convergent in $L^{2}$. Once again, denoting this subsequence by $V_{i}$, we have 
$V_{i} \stackrel{L^{2}}{\rightarrow} \tilde{V}$. But then $V_{i} \stackrel{L^{2}}{\rightarrow} \tilde{V}$ so

$$
0=\left\langle V_{i}, \operatorname{ker} \mathfrak{D}^{\phi}\right\rangle_{L^{2}} \rightarrow\left\langle\tilde{V}, \operatorname{ker} \mathfrak{D}^{\phi}\right\rangle_{L^{2}}
$$

Hence $V_{i} \stackrel{L^{2}}{\rightarrow} \tilde{V} \in\left(\operatorname{ker} \mathfrak{D}^{\phi}\right)^{\perp_{L^{2}}}$.

But $\iota: H^{1} \hookrightarrow L^{2}$ is continuous, so $V_{i} \stackrel{H^{1}}{\rightarrow} V \Rightarrow \iota V_{i} \stackrel{L^{2}}{\rightarrow} \iota V$, and so $\tilde{V}=V$ by uniqueness of weak limits. So $V \in \operatorname{ker} \mathfrak{D}^{\phi}$ and $V \in\left(\operatorname{ker} \mathfrak{D}^{\phi}\right)^{\perp} L^{2}$, and hence $V=0$, so $V_{i} \stackrel{L^{2}}{\rightarrow} 0$. Then, by (3.6) and (3.8),

$$
\begin{aligned}
1 & =\left\|V_{i}\right\|_{H^{1}}^{2}=\left\|V_{i}\right\|_{L^{2}}^{2}+\sum_{\alpha}\left\|\nabla_{E_{\alpha}}^{\phi} V_{i}\right\|_{L^{2}}^{2} \\
& =2 \operatorname{Hess}_{\phi}\left(V_{i}, V_{i}\right)+\left\|V_{i}\right\|_{L^{2}}^{2}+\sum_{\alpha} 2\left\langle R^{N}\left(d \phi E_{\alpha}, V_{i}\right) d \phi E_{\alpha}, V_{i}\right\rangle_{L^{2}} \\
& \leq 2 \operatorname{Hess}_{\phi}\left(V_{i}, V_{i}\right)+\left\|V_{i}\right\|_{L^{2}}^{2}+C_{\phi}\left\|V_{i}\right\|_{L^{2}}^{2}
\end{aligned}
$$

where $C_{\phi}>0$ is a constant, by compactness of $N$ and the tensorial property of $\mathfrak{R}^{N}$. Taking limits of both sides of (3.13) and using $\tau(\phi)=0$ and $V_{i} \stackrel{L^{2}}{\rightarrow} 0$, one sees that $1 \leq 0$, a contradiction.

We remark that the $L^{2}$ version of this result, that there exists a constant $\tau(\phi)>0$ such that $\operatorname{Hess}_{\phi}(V, V) \geq \tau(\phi)\|V\|_{L^{2}}^{2}$ for all $V \perp_{L^{2}}$ ker $\mathfrak{J}^{\phi}$, is much easier to prove. Since $\mathfrak{J}^{\phi}$ is elliptic, self adjoint and positive definite (for weakly stable $\phi$ ), and $M$ is compact, we know immediately that the spectrum of $\mathfrak{J}^{\phi}$ is discrete and, normal to its kernel, bounded away from 0 . The result immediately follows. In fact the optimal constant $\tau_{L^{2}}(\phi)$ in this case is just the lowest nonvanishing eigenvalue of $\mathfrak{J}^{\phi}$. Hence the optimal bound is attained in this case, by any eigensection with this eigenvalue. We remark also that the spectrum of $\mathfrak{J}^{\phi}$ is of independent physical interest since it gives the semiclassical meson spectrum of the sigma model in the topological sector $[\phi]$.

\section{Continuity of $\tau(\phi)$}

Let $\phi_{n}: M \rightarrow N$ be a sequence of smooth holomorphic maps between compact Kähler manifolds, converging in $C^{1}$ to a smooth holomorphic map $\phi: M \rightarrow N$. From Theorem 3] we have for each $n$ a positive constant $\tau\left(\phi_{n}\right)$ and the positive constant $\tau(\phi)$, which give lower bounds for the ratio $\operatorname{Hess}(V, V) /\langle V, V\rangle_{H_{1}}$ for any $H^{1}$ section of the pullback bundle $L^{2}$ orthogonal to all Jacobi fields. In this section we will establish conditions sufficient to guarantee that $\lim _{n \rightarrow \infty} \tau\left(\phi_{n}\right)=\tau(\phi)$. In subsequent sections we will show that these conditions are met in the cases of interest to us.

In order to compare various quantities (especially the Hessian and $H^{1}$ norm) at different maps, it is convenient to make various identifications so that we can treat all geometric quantities and operators as being defined on the fixed bundle $E=\phi^{*} T N$. Since $\phi_{n} \rightarrow \phi$, for sufficiently large $n$ each pullback bundle $E_{n}=\phi_{n}^{*} T N$ is topologically equivalent to $E$. However, since each bundle $E_{n}$ comes naturally equipped with both an inner product and a 
compatible connexion (both of which occur in the Hessian and the $H^{1}$-norm of a section) we would also like to transfer these geometric structures to the bundle $E$.

Again since $\phi_{n} \rightarrow \phi$, for each $x \in M$ (and for each sufficiently large $n$ ) there is a unique minimizing geodesic joining $\phi_{n}(x)$ to $\phi(x)$. By parallel transporting vectors along this unique geodesic we construct a canonical isometry between the fibres of $E$ and the fibres of $E_{n}$ at each point $x \in M$, and hence a natural $L^{2}$ isometry between $E$ and $E_{n}$. Using this isometry we can interpret the natural connexion on each $E_{n}$ as a connexion on $E$, which we shall write $\nabla_{n}$. Using the connexion $\nabla_{n}$ and the (fixed) $L^{2}$ metric on $E$ we can now interpret each of the Jacobi operators $\mathfrak{J}^{\phi_{n}}$ (and hence also the associated symmetric bilinear form Hess ${ }_{\phi_{n}}$ ) as an operator on sections of the fixed bundle $E$. Similarly, for each $n$ we get an $H_{\phi_{n}}^{1}$ norm on sections of $E$, by using the connection $\nabla_{n}$ and the fixed $L^{2}$ metric. Since the difference of any two connexions on $E$ is tensorial, it is clear that for each $n$ (sufficiently large) the $H_{\phi_{n}}^{1}$ norm is equivalent to the $H_{\phi}^{1}$ norm on sections of $E$.

Suppose now that the difference between the connexions $\nabla_{n}$ and $\nabla$ tends to zero in the the following pointwise sense

$$
\left|\nabla V-\nabla_{n} V\right| \leq a_{n}|V|
$$

where each $a_{n}$ is a positive number, $a_{n} \rightarrow 0$ as $n \rightarrow \infty$, and $|$.$| refers to the natural pointwise$ norms on the bundles $E \otimes T^{*} M$ and $E$, and $V$ is a smooth section of $E$.

Lemma 4 Suppose that (4.1) holds, then the following inequalities also hold:

(i) | $\|\nabla V\|^{2}-\left\|\nabla_{n} V\right\|^{2} \mid \leq b_{n}\|V\|_{1}^{2}$

(ii) | $\|V\|_{1}^{2}-\|V\|_{H_{\phi_{n}}^{1}}^{2} \mid \leq b_{n}\|V\|_{1}^{2}$

(iii) $\left|\operatorname{Hess}_{\phi}(V, V)-\operatorname{Hess}_{\phi_{n}}(V, V)\right| \leq c_{n}\|V\|_{1}^{2}$

where $b_{n}, c_{n}$ are positive numbers which tend to zero as $n \rightarrow \infty$, and $\|$. $\|$ is the $L^{2}$ norm on $\Gamma(E),\|.\|_{1}$ is the $H^{1}$ norm on $\Gamma(E)$ defined using the connection $\nabla$ and \|\|$_{H_{\phi_{n}}^{1}}$ is the $H^{1}$ norm on $\Gamma(E)$ defined using the connection $\nabla_{n}$.

Proof: (i) Elementary manipulations involving the triangle inequality, inequality (4.1) and the Cauchy-Schwarz inequality yield the following chain of inequalities

$$
\begin{aligned}
\left|\int_{M}\left(|\nabla V|^{2}-\left|\nabla_{n} V\right|^{2}\right)\right| & \leq\left.\int_{M}|| \nabla V\right|^{2}-\left|\nabla_{n} V\right|^{2} \mid \\
& \leq \int_{M}|| \nabla V|-| \nabla_{n} V|| \cdot|| \nabla V|+| \nabla_{n} V|| \\
& \leq \int_{M}|| \nabla V|-| \nabla_{n} V|| \cdot\left(|2| \nabla V|+| \nabla V-\nabla_{n} V||\right) \\
& \leq 2 a_{n} \int_{M}|V||\nabla V|+a_{n}^{2} \int_{M}|V|^{2} \\
& \leq 2 a_{n}\||V|\| \cdot\||\nabla V|\|+a_{n}^{2}\|V\|^{2} \\
& \leq\left(2 a_{n}+a_{n}^{2}\right)\|V\|_{1}^{2} .
\end{aligned}
$$

Hence the result follows with $b_{n}=a_{n}\left(2+a_{n}\right)$.

(ii) This is immediate from the definition of the $H^{1}$ norm and part (i). 
(iii) The definition of $\mathfrak{R}^{\phi}$ (see equation 3.7), together with the compactness of $M$ and the fact that $\phi_{n} \rightarrow \phi$ in $C^{1}$ implies that

$$
\lim _{n \rightarrow \infty}\left\|\mathfrak{R}^{\phi}-\mathfrak{R}^{\phi_{n}}\right\|=0 .
$$

Using part (i) and the definition of Hess we have

$$
\begin{aligned}
\left|\operatorname{Hess}_{\phi}(V, V)-\operatorname{Hess}_{\phi_{n}}(V, V)\right| & =\left|\frac{1}{2}\|\nabla V\|^{2}-\frac{1}{2}\left\|\nabla_{n} V\right\|^{2}-\left\langle\mathfrak{R}^{\phi} V, V\right\rangle+\left\langle\mathfrak{R}^{\phi_{n}} V, V\right\rangle\right| \\
& \leq \frac{1}{2}\left|\|\nabla V\|^{2}-\left\|\nabla_{n} V\right\|^{2}\right|+\left|\left\langle\mathfrak{R}^{\phi} V, V\right\rangle-\left\langle\mathfrak{R}^{\phi_{n}} V, V\right\rangle\right| \\
& \leq b_{n}\|V\|_{1}^{2}+\left\|\left(\mathfrak{R}^{\phi}-\mathfrak{R}^{\phi_{n}}\right) V\right\|\|V\| \\
& \leq b_{n}\|V\|_{1}^{2}+\left\|\mathfrak{R}^{\phi}-\mathfrak{R}^{\phi_{n}}\right\|\|V\|^{2} .
\end{aligned}
$$

The result now follows from (4.2).

We will also need the following simple proposition on the continuity of $L^{2}$ orthogonal projection operators.

Proposition 5 Let $H^{k}(E)$ denote the $H^{k}$ sections of $E$. Let $V_{n}$ be a sequence of finite dimensional vector subspaces of $H^{k}(E)$ of constant dimension $p$ which converge to another $p$ dimensional subspace $V$ of $H^{k}(E)$, in the following sense: there exists an $L^{2}$-orthonormal basis $e_{1}^{n}, \ldots, e_{p}^{n}$ of $V_{n}$ and an $L^{2}$-orthonormal basis $e_{1}, \ldots, e_{p}$ of $V$ so that for each $i=1, \ldots p$, $e_{i}^{n} \rightarrow e_{i}$ in $H^{k}$ as $n \rightarrow \infty$. Denote by $P_{n}$ and $P$ the $L^{2}$ orthogonal projections onto $V_{n}^{\perp}$ and $V^{\perp}$ respectively. Then $P_{n} \rightarrow P$ in the operator norm on $B\left(H^{k}(E), H^{k}(E)\right)$, the bounded linear maps between $H^{k}(E)$ and itself.

Proof: In this proof, norms and inner products without subscripts will refer to $L^{2}$ norms and inner products, while $H^{k}$ norms and inner products will be referred to with the subscript $k$. Since $P_{n} v=v-\sum_{i}\left\langle v, e_{i}^{n}\right\rangle e_{i}^{n}$ and $P v=v-\sum_{i}\left\langle v, e_{i}\right\rangle e_{i}$ we have

$$
\begin{aligned}
\left\|P_{n} v-P v\right\|_{k} & =\left\|\sum_{i}\left\langle v, e_{i}^{n}\right\rangle e_{i}^{n}-\sum_{i}\left\langle v, e_{i}\right\rangle e_{i}\right\|_{k} \leq \sum_{i}\left\|\left\langle v, e_{i}^{n}\right\rangle e_{i}^{n}-\left\langle v, e_{i}\right\rangle e_{i}\right\|_{k} \\
& \leq \sum_{i}\left\|\left\langle v, e_{i}^{n}\right\rangle e_{i}^{n}-\left\langle v, e_{i}^{n}\right\rangle e_{i}+\left\langle v, e_{i}^{n}\right\rangle e_{i}-\left\langle v, e_{i}\right\rangle e_{i}\right\|_{k} \\
& \leq \sum_{i}\left|\left\langle v, e_{i}^{n}\right\rangle\right|\left\|e_{i}^{n}-e_{i}\right\|_{k}+\left\|e_{i}\right\|_{k}\left|\left\langle v, e_{i}^{n}-e_{i}\right\rangle\right| \\
& \leq\|v\|\left(\sum_{i}\left\|e_{i}^{n}\right\|\left\|e_{i}^{n}-e_{i}\right\|_{k}+\left\|e_{i}\right\|_{k}\left\|e_{i}^{n}-e_{i}\right\|\right) \\
& \leq\|v\|\left(\sum_{i}\left(1+\left\|e_{i}\right\|_{k}\right)\left\|e_{i}^{n}-e_{i}\right\|_{k}\right) .
\end{aligned}
$$

So for all $v \neq 0 \in H^{k}$ we have,

$$
\frac{\left\|\left(P_{n}-P\right) v\right\|_{k}}{\|v\|_{k}} \leq \frac{\left\|\left(P_{n}-P\right) v\right\|_{k}}{\|v\|} \leq\left(\sum_{i}\left(1+\left\|e_{i}\right\|_{k}\right)\left\|e_{i}^{n}-e_{i}\right\|_{k}\right) .
$$


Since by assumption $e_{i}^{n} \rightarrow e_{i}$ strongly in $H^{k}$ for each $i$, (4.4) implies that

$$
\lim _{n \rightarrow \infty} \sup _{v \neq 0} \frac{\left\|\left(P_{n}-P\right) v\right\|_{k}}{\|v\|_{k}}=0
$$

as required.

We now state the main result of this section, a theorem giving sufficient conditions for $\tau(\phi)$ to depend continuously on the holomorphic map $\phi$. The analogous result in Stuart's analysis of slowly moving abelian Higgs vortices is Lemma 3.2 of [23]. Our proof of Theorem [6 is inspired by Stuart's argument.

Theorem 6 Let $\phi_{n}: M \rightarrow N$ be a sequence of smooth holomorphic maps between compact Kähler manifolds converging in $C^{1}$ to the smooth holomorphic map $\phi: M \rightarrow N$. Suppose all the Jacobi fields of $\phi$ are integrable and that the conclusions of Lemma 4 hold. Then

$$
\lim _{n \rightarrow \infty} \tau\left(\phi_{n}\right)=\tau(\phi)
$$

Proof: Let $P_{n}, P: H^{1}(E) \rightarrow H^{1}(E)$ denote $L^{2}$ orthogonal projection onto ker $\mathfrak{D}^{\phi_{n}}{ }^{\perp}$ and ker $\mathfrak{D}^{\phi^{\perp}}$ respectively. Since $\phi$ is Jacobi integrable, the space of sufficiently $C^{1}$-close holomorphic maps is a $C^{\infty}$ manifold of dimension equal to dim ker $\mathfrak{D}^{\phi}$. This also holds for all $\phi_{n}$ for all $n$ sufficiently large. In particular, the subspaces ker $\mathfrak{D}^{\phi_{n}}$ satisfy the hypotheses of the previous proposition for any $k$, and in particular for $k=1$. Hence by the previous proposition we have

$$
\left\|P-P_{n}\right\|_{1}=d_{n}
$$

for some positive numbers $d_{n}$ tending to 0 as $n \rightarrow \infty$.

We will prove (i) $\tau(\phi) \geq \lim \sup _{n \rightarrow \infty} \tau\left(\phi_{n}\right)$ and (ii) $\tau(\phi) \leq \liminf _{n \rightarrow \infty} \tau\left(\phi_{n}\right)$. From (i) and (ii) it follows that $\lim _{n \rightarrow \infty} \tau\left(\phi_{n}\right)$ exists and equals $\tau(\phi)$.

Proof of (i): consider for any $V \in H^{1}(E) \cap\left(\operatorname{ker} \mathfrak{D}^{\phi}\right)^{\perp}$, the section $V_{n}:=P_{n} V \in H^{1}(E) \cap$ $\left(\text { ker } \mathfrak{D}^{\phi_{n}}\right)^{\perp}$. Since $\left\|V-V_{n}\right\|_{H_{\phi}^{1}}=\left\|\left(P-P_{n}\right) V\right\|_{H_{\phi}^{1}} \leq d_{n}\|V\|_{H_{\phi}^{1}}$ we get

$$
\left|\left\langle V_{n}, V_{n}\right\rangle_{H_{\phi}^{1}}-\langle V, V\rangle_{H_{\phi}^{1}}\right| \leq\left\|V-V_{n}\right\|_{H_{\phi}^{1}}\left(\|V\|_{H_{\phi}^{1}}+\left\|V_{n}\right\|_{H_{\phi}^{1}}\right) \leq d_{n}\left(d_{n}+2\right)\langle V, V\rangle_{H_{\phi}^{1}} .
$$

Using the previous inequality and inequality (ii) of Lemma 4 we have

$$
\begin{aligned}
\left|\langle V, V\rangle_{H_{\phi}^{1}}-\left\langle V_{n}, V_{n}\right\rangle_{H_{\phi_{n}}^{1}}\right| & \leq\left|\left\langle V_{n}, V_{n}\right\rangle_{H_{\phi_{n}}^{1}}-\left\langle V_{n}, V_{n}\right\rangle_{H_{\phi}^{1}}\right|+\left|\left\langle V_{n}, V_{n}\right\rangle_{H_{\phi}^{1}}-\langle V, V\rangle_{H_{\phi}^{1}}\right| \\
& \leq b_{n}\left\langle V_{n}, V_{n}\right\rangle_{H_{\phi}^{1}}+d_{n}\left(d_{n}+2\right)\langle V, V\rangle_{H_{\phi}^{1}} \\
& \leq f_{n}\langle V, V\rangle_{H_{\phi}^{1}}
\end{aligned}
$$

where $f_{n}:=\left(b_{n}+d_{n}\left(d_{n}+2\right)\left(1+b_{n}\right)\right)$ is another sequence of positive numbers tending to zero as $n \rightarrow \infty$. 
Since $V=V_{n}+K_{n}$ for some $K_{n} \in \operatorname{ker} \mathfrak{D}^{\phi_{n}}$, we have $\operatorname{Hess}_{\phi_{n}}\left(V_{n}, V_{n}\right)=\operatorname{Hess}_{\phi_{n}}(V, V)$. Hence

$$
\begin{aligned}
& \left|\frac{\operatorname{Hess}_{\phi}(V, V)}{\langle V, V\rangle_{H_{\phi}^{1}}}-\frac{\operatorname{Hess}_{\phi_{n}}\left(V_{n}, V_{n}\right)}{\left\langle V_{n}, V_{n}\right\rangle_{H_{\phi_{n}}^{1}}}\right|=\left|\frac{\operatorname{Hess}_{\phi}(V, V)}{\langle V, V\rangle_{H_{\phi}^{1}}}-\frac{\operatorname{Hess}_{\phi_{n}}(V, V)}{\left\langle V_{n}, V_{n}\right\rangle_{H_{\phi_{n}}^{1}}}\right| \\
& \leq \frac{\operatorname{Hess}_{\phi}(V, V)\left|\langle V, V\rangle_{H_{\phi}^{1}}-\left\langle V_{n}, V_{n}\right\rangle_{H_{\phi_{n}}^{1}}\right|+\langle V, V\rangle_{H_{\phi}^{1}}\left|\operatorname{Hess}_{\phi}(V, V)-\operatorname{Hess}_{\phi_{n}}(V, V)\right|}{\langle V, V\rangle_{H_{\phi}^{1}}\left\langle V_{n}, V_{n}\right\rangle_{H_{\phi_{n}}^{1}}} \\
& \leq \frac{f_{n} \operatorname{Hess}_{\phi}(V, V)+c_{n}\langle V, V\rangle_{H_{\phi}^{1}}}{\langle V, V\rangle_{H_{\phi_{n}}^{1}}} \leq \frac{f_{n}}{1-f_{n}} \frac{\operatorname{Hess}_{\phi}(V, V)}{\langle V, V\rangle_{H_{\phi}^{1}}}+\frac{c_{n}}{1-f_{n}} .
\end{aligned}
$$

Let $\left\{V^{j}\right\}_{j=1}^{\infty} \in H^{1}(E) \cap\left(\operatorname{ker} \mathfrak{D}^{\phi}\right)^{\perp}$ be a minimizing sequence for $\tau(\phi)$ with unit $H_{\phi}^{1}$ norm, that is

$$
\lim _{j \rightarrow \infty} \frac{\operatorname{Hess}_{\phi}\left(V^{j}, V^{j}\right)}{\left\langle V^{j}, V^{j}\right\rangle_{H_{\phi}^{1}}}=\lim _{j \rightarrow \infty} \operatorname{Hess}_{\phi}\left(V^{j}, V^{j}\right)=\tau(\phi) .
$$

Hence there exists a positive constant $C$ and numbers $\epsilon_{j}$ tending to zero as $j \rightarrow \infty$, such that

$$
\operatorname{Hess}_{\phi}\left(V^{j}, V^{j}\right)=\tau(\phi)+\epsilon_{j}
$$

and

$$
\operatorname{Hess}_{\phi_{n}}\left(V^{j}, V^{j}\right) \leq C
$$

for all $j$ and $n$. Define $V_{n}^{j}=P_{n} V^{j} \in H^{1}(E) \cap\left(\operatorname{ker} \mathfrak{D}^{\phi_{n}}\right)^{\perp}$. The two previous facts together with (4.8) imply that

$$
\left|\tau(\phi)+\epsilon_{j}-\frac{\operatorname{Hess}_{\phi_{n}}\left(V_{n}^{j}, V_{n}^{j}\right)}{\left\langle V_{n}^{j}, V_{n}^{j}\right\rangle_{H_{\phi_{n}}^{1}}}\right| \leq \frac{C f_{n}+c_{n}}{1-f_{n}}
$$

holds for all $j$ and $n$. For any fixed $n$, taking the limit as $j \rightarrow \infty$ we see that

$$
\tau\left(\phi_{n}\right) \leq \tau(\phi)+\frac{C f_{n}+c_{n}}{1-f_{n}}
$$

Hence for any limit point $l$ of the sequence $\left\{\tau\left(\phi_{n}\right)\right\}_{n=1}^{\infty}$ we have $l \leq \tau(\phi)$. In particular we have

$$
\limsup _{n \rightarrow \infty} \tau\left(\phi_{n}\right) \leq \tau(\phi)
$$

as required.

Proof of (ii): Since the proof of (ii) is very similar in character to the proof of (i) we shall omit some details. Consider the projection $P V_{n}$ of an element $V_{n} \in H^{1}(E) \cap\left(\operatorname{ker} \mathfrak{D}^{\phi_{n}}\right)^{\perp}$ into $H^{1}(E) \cap\left(\operatorname{ker} \mathfrak{D}^{\phi}\right)^{\perp}$. Several applications of the triangle inequality, together with the inequalities in Lemma 4 and the fact that $P_{n} \rightarrow P$ in $H^{1}$, show that there exist two sequences of positive numbers $\left\{g_{n}\right\}$ and $\left\{h_{n}\right\}$ with $\lim _{n \rightarrow \infty} g_{n}=\lim _{n \rightarrow \infty} h_{n}=0$ so that

$$
\left|\left\langle V_{n}, V_{n}\right\rangle_{H_{\phi_{n}}^{1}}-\left\langle P V_{n}, P V_{n}\right\rangle_{H_{\phi}^{1}}\right| \leq g_{n}\left\langle P V_{n}, P V_{n}\right\rangle_{H_{\phi}^{1}}
$$


and

$$
\left|\left\langle V_{n}, V_{n}\right\rangle_{H_{\phi}^{1}}-\left\langle P V_{n}, P V_{n}\right\rangle_{H_{\phi}^{1}}\right| \leq h_{n}\left\langle V_{n}, V_{n}\right\rangle_{H_{\phi}^{1}}
$$

hold for any $V_{n} \in H^{1}(E) \cap\left(\operatorname{ker} \mathfrak{D}^{\phi_{n}}\right)^{\perp}$. These two inequalities, combined with the inequalities of Lemma 4 and the fact that $\operatorname{Hess}_{\phi}\left(P V_{n}, P V_{n}\right)=\operatorname{Hess}_{\phi}\left(V_{n}, V_{n}\right)$ prove that

$$
\begin{aligned}
& \left|\frac{\operatorname{Hess}_{\phi}\left(P V_{n}, P V_{n}\right)}{\left\langle P V_{n}, P V_{n}\right\rangle_{H_{\phi}^{1}}}-\frac{\operatorname{Hess}_{\phi_{n}}\left(V_{n}, V_{n}\right)}{\left\langle V_{n}, V_{n}\right\rangle_{H_{\phi_{n}}^{1}}}\right|=\left|\frac{\operatorname{Hess}_{\phi}\left(V_{n}, V_{n}\right)}{\left\langle P V_{n}, P V_{n}\right\rangle_{H_{\phi}^{1}}}-\frac{\operatorname{Hess}_{\phi_{n}}\left(V_{n}, V_{n}\right)}{\left\langle V_{n}, V_{n}\right\rangle_{H_{\phi_{n}}^{1}}}\right| \\
& \leq \frac{\operatorname{Hess}_{\phi_{n}}\left(V_{n}, V_{n}\right)\left|\left\langle V_{n}, V_{n}\right\rangle_{H_{\phi_{n}}^{1}}-\left\langle P V_{n}, P V_{n}\right\rangle_{H_{\phi}^{1}}\right|+\left\langle V_{n}, V_{n}\right\rangle_{H_{\phi_{n}}^{1}}\left|\operatorname{Hess}_{\phi}\left(V_{n}, V_{n}\right)-\operatorname{Hess}_{\phi_{n}}\left(V_{n}, V_{n}\right)\right|}{\left\langle V_{n}, V_{n}\right\rangle_{H_{\phi_{n}}^{1}}\left\langle P V_{n}, P V_{n}\right\rangle_{H_{\phi}^{1}}} \\
& \leq \frac{g_{n} \operatorname{Hess}_{\phi_{n}}\left(V_{n}, V_{n}\right)\left\langle P V_{n}, P V_{n}\right\rangle_{H_{\phi}^{1}}+c_{n}\left\langle V_{n}, V_{n}\right\rangle_{H_{\phi_{n}}^{1}}\left\langle V_{n}, V_{n}\right\rangle_{H_{\phi}^{1}}}{\left\langle V_{n}, V_{n}\right\rangle_{H_{\phi_{n}}^{1}}\left\langle P V_{n}, P V_{n}\right\rangle_{H_{\phi}^{1}}} \\
& \leq g_{n} \frac{\operatorname{Hess}_{\phi_{n}}\left(V_{n}, V_{n}\right)}{\left\langle V_{n}, V_{n}\right\rangle_{H_{\phi_{n}}}}+c_{n} \frac{\left\langle V_{n}, V_{n}\right\rangle_{H_{\phi}^{1}}}{\left\langle P V_{n}, P V_{n}\right\rangle_{H_{\phi}^{1}}} \leq g_{n} \frac{\operatorname{Hess}_{\phi_{n}}\left(V_{n}, V_{n}\right)}{\left\langle V_{n}, V_{n}\right\rangle_{H_{\phi_{n}}^{1}}}+\frac{c_{n}}{1-h_{n}}
\end{aligned}
$$

holds for any $V_{n} \in H^{1}(E) \cap\left(\operatorname{ker} \mathfrak{D}^{\phi_{n}}\right)^{\perp}$.

Let $\left\{V_{n}^{j}\right\}_{j=1}^{\infty} \in H^{1}(E) \cap\left(\operatorname{ker} \mathfrak{D}^{\phi_{n}}\right)^{\perp}$ be a minimizing sequence for $\tau\left(\phi_{n}\right)$ with unit $H_{\phi_{n}}^{1}$ norm, that is

$$
\lim _{j \rightarrow \infty} \frac{\operatorname{Hess}_{\phi_{n}}\left(V_{n}^{j}, V_{n}^{j}\right)}{\left\langle V_{n}^{j}, V_{n}^{j}\right\rangle_{H_{\phi_{n}}^{1}}^{1}}=\lim _{j \rightarrow \infty} \operatorname{Hess}_{\phi_{n}}\left(V_{n}^{j}, V_{n}^{j}\right)=\tau\left(\phi_{n}\right) .
$$

Hence there exists a positive constant $C$ and numbers $\epsilon_{n}^{j}$ tending to zero as $j \rightarrow \infty$, such that

$$
\operatorname{Hess}_{\phi}\left(V_{n}^{j}, V_{n}^{j}\right)=\tau\left(\phi_{n}\right)+\epsilon_{n}^{j}
$$

and

$$
\operatorname{Hess}_{\phi}\left(V_{n}^{j}, V_{n}^{j}\right) \leq C
$$

for all $j$ and $n$. Consider the sequence $\widetilde{V}_{n}^{j}=P V_{n}^{j} \in H^{1}(E) \cap\left(\operatorname{ker} \mathfrak{D}^{\phi}\right)^{\perp}$. The two previous facts together with (4.11) imply that

$$
\left|\frac{\operatorname{Hess}_{\phi}\left(\widetilde{V}_{n}^{j}, \widetilde{V}_{n}^{j}\right)}{\left\langle\widetilde{V}_{n}^{j}, \widetilde{V}_{n}^{j}\right\rangle_{H_{\phi}^{1}}}-\left(\tau(\phi)+\epsilon_{n}^{j}\right)\right| \leq C g_{n}+\frac{c_{n}}{1-h_{n}}
$$

holds for all $j$ and $n$. For any fixed $n$, taking the limit as $j \rightarrow \infty$ we see that

$$
\tau(\phi) \leq \tau\left(\phi_{n}\right)+C g_{n}+\frac{c_{n}}{1-h_{n}} .
$$

Hence for any limit point $l$ of the sequence $\left\{\tau\left(\phi_{n}\right)\right\}_{n=1}^{\infty}$ we have $\tau(\phi) \leq l$. In particular

$$
\tau(\phi) \leq \liminf _{n \rightarrow \infty} \tau\left(\phi_{n}\right)
$$

as claimed. 


\section{The case of Rat}

It is interesting to consider the simplest nontrivial case, namely $\mathrm{M}_{1}=\operatorname{Hol}_{1}\left(S^{2}, S^{2}\right)=\operatorname{Rat}_{1}$, in detail. Although $M=N=S^{2}$ in this case, it is often helpful to distinguish between domain and codomain by continuing to denote them $M, N$ respectively. We shall do this when clarity requires. The $G$ action on $\mathrm{M}_{n}$ introduced in section 2 extends naturally to $C^{\infty}\left(S^{2}, S^{2}\right)$, and leaves $E[\phi]$ invariant. It follows that $\tau(\phi)$ is constant on $G$ orbits in $\mathrm{M}_{n}$. Since $G$ acts with cohomogeneity 1 on $\mathrm{M}_{1}$, it suffices to consider $\tau(\phi)$ for the one-parameter family of maps

$$
\phi_{\mu}: z \mapsto \mu z, \quad \mu \in[1, \infty)
$$

lying on the curve $\Gamma=\left\{\left(\left[\mathbb{I}_{2}\right],(0,0, \lambda)\right): \lambda \geq 0\right\}$ in $\mathrm{M}_{1}$ (so $\mu=\left(\sqrt{1+\lambda^{2}}+\lambda\right)^{2}$ as in section 2). We may think of $\tau$ as a positive function $\tau(\mu)$ on $[1, \infty)$. We will first use the results of section 4 to prove that $\tau(\mu)$ is continuous. Positivity and continuity of $\tau$ ensure that it is bounded away from zero on any compact set. The domain of $\tau$ (whether thought of as a function on $\mathrm{M}_{1}$ or on $\left.[1, \infty)\right)$ is noncompact, however, so we cannot conclude that $\tau$ is globally bounded away from zero. The essential question is, then, how does $\tau(\mu)$ behave as $\mu \rightarrow \infty$, that is, as the lump collapses to zero width? We will prove that $\tau(\mu) \rightarrow 0$.

First we address the issue of continuity. By symmetry, it suffices to consider a sequence of degree 1 holomorphic maps $\phi_{n}$ in the curve $\Gamma$, labelled by a sequence $\mu_{n}$ in $[1, \infty)$. If $\mu_{n} \rightarrow \hat{\mu}$ then the corresponding maps $\phi_{n}: z \mapsto \mu_{n} z$ converge in $C^{1}$ to $\hat{\phi}: z \mapsto \hat{\mu} z$. Since every holomorphic map $S^{2} \rightarrow S^{2}$ is Jacobi integrable, we may apply the results of section 4 . We merely need to prove that the pointwise inequality (4.1) on the pullback connexions $\nabla_{n}\left(\nabla^{\phi_{n}}\right.$ transfered to $\hat{\phi}^{*} T N$ ) holds for any such sequence $\mu_{n}$. Then Lemma 4 applies, and continuity of $\tau$ follows from Theorem 6 .

We must first construct the canonical isometry between each bundle $\phi_{n}^{*} T N$ and the fixed bundle $\hat{\phi}^{*} T N$ equipped with their $L^{2}$ inner products. To this end, it is convenient to define an orthonormal frame $E_{1}=\partial / \partial \theta, E_{2}=\operatorname{cosec} \theta \partial / \partial \varphi$ on $S^{2}$, where $(\theta, \varphi)$ are the usual polar coordinates, and the corresponding sections $\widetilde{E}_{i}^{n}=E_{i} \circ \phi_{n} \in \Gamma\left(\phi_{n}^{*} T N\right), \hat{E}_{i}=E_{i} \circ \phi \in \Gamma\left(\phi^{*} T N\right)$, $i=1,2$. Note that varying $\phi$ within the family $\Gamma$ sends each fixed $p \in M$ along a geodesic of constant $\varphi$ in $N$. Note also that the frame $E_{1}, E_{2}$ is parallel along geodesics of constant $\varphi$, so the canonical isometry between $\phi_{n}^{*} T N$ and $\hat{\phi}^{*} T N$ is simply given by the identification

$$
\widetilde{E}_{1}^{n} \equiv \hat{E}_{1}, \quad \widetilde{E}_{2}^{n} \equiv \hat{E}_{2} .
$$

It is this property which makes polar coordinates particularly natural for our purposes.

In this coordinate system, a holomorphic map $\phi: z \mapsto \mu z$ is

$$
(\theta, \varphi) \mapsto\left(f_{\mu}(\theta), \varphi\right), \quad f_{\mu}(\theta)=2 \cot ^{-1}\left(\mu \cot \frac{\theta}{2}\right)
$$

We will construct the pullback connexion $\nabla^{\phi}$ by computing its action on $\widetilde{E}_{i}=E_{i} \circ \phi, i=1,2$. The Levi-Civita connexion on $S^{2}$ is

$$
\nabla E_{1}=\cot \theta e_{2} \otimes E_{2}, \quad \nabla E_{2}=-\cot \theta e_{2} \otimes E_{1}
$$


or equivalently,

$$
\nabla e_{1}=\cot \theta e_{2} \otimes e_{2}, \quad \nabla e_{2}=-\cot \theta e_{2} \otimes e_{1},
$$

where $e_{1}, e_{2}$ is the coframe dual to $E_{1}, E_{2}$. The following properties of $\nabla^{\phi}$ are essential for computations:

(a) if $u \in T_{p} M, Y \in \Gamma\left(\phi^{*} T N\right)$ and $g \in C^{\infty}(M)$, then $\nabla_{u}^{\phi} f Y=u[f] Y+f \nabla_{u}^{\phi} Y$;

(b) if $Y \in \Gamma\left(\phi^{*} T N\right)$ may locally be identified with a vector field $\widetilde{Y}$ on $N$ (i.e. $Y=\widetilde{Y} \circ \phi$, on a neighbourhood of $p$ ), then $\nabla_{u}^{\phi} Y=\nabla_{d \phi u}^{N} \widetilde{Y}$.

Now $d \phi E_{1}=f_{\mu}^{\prime} \widetilde{E}_{1}$ and $d \phi E_{2}=\operatorname{cosec} \theta \sin f_{\mu} \widetilde{E}_{2}$, where ' denotes differentiation with respect to $\theta$. Hence

$$
\begin{gathered}
\nabla_{E_{1}}^{\phi} \widetilde{E}_{1}=\nabla_{E_{1}}^{\phi} \widetilde{E}_{2}=0 \\
\nabla_{E_{2}}^{\phi} \widetilde{E}_{1}=\frac{\sin f_{\mu}}{\sin \theta} \nabla_{\widetilde{E}_{2}} \widetilde{E}_{1}=\frac{\sin f_{\mu}}{\sin \theta} \cot f_{\mu} \widetilde{E}_{2}=\frac{\cos f_{\mu}}{\sin \theta} \widetilde{E}_{2} \\
\nabla_{E_{2}}^{\phi} \widetilde{E}_{2}=\frac{\sin f_{\mu}}{\sin \theta} \nabla_{\widetilde{E}_{2}} \widetilde{E}_{2}=-\frac{\sin f_{\mu}}{\sin \theta} \cot f_{\mu} \widetilde{E}_{2}=-\frac{\cos f_{\mu}}{\sin \theta} \widetilde{E}_{2} .
\end{gathered}
$$

Note that when $\mu=1, \phi=\mathrm{Id}$, and $\nabla^{\mathrm{Id}}=\nabla$, so (5.6) should reduce to (5.4), which it does.

Any section of $\phi^{*} T N$ may be written

$$
W=V+J^{N} U, \quad V=\mathcal{V}(\theta, \varphi) \widetilde{E}_{1}, \quad U=\mathcal{U}(\theta, \varphi) \widetilde{E}_{1} .
$$

Note that $\nabla^{\phi}$ commutes with $J^{N}$ by the Kähler property. By the defining property (a), above, and (5.6), one sees that

$$
\nabla^{\phi} V=\mathcal{V}_{\theta} e_{1} \otimes \widetilde{E}_{1}+\frac{1}{\sin \theta} \mathcal{V}_{\varphi} e_{2} \otimes \widetilde{E}_{2}+\mathcal{V} \frac{\cos f_{\mu}}{\sin \theta} e_{2} \otimes \widetilde{E}_{2}
$$

where subscripts $\theta, \varphi$ denote partial derivatives. This gives us explicit formulae for $\nabla^{\hat{\phi}}$ and $\nabla^{\phi_{n}}$. Using the identification (5.2) we may transfer a section $W$ of $\hat{\phi}^{*} T N$ to $\phi_{n}^{*} T N$, act with $\nabla^{\phi_{n}}$, then transfer back using the same identification, calling the result $\nabla_{n} W$. The difference between $\nabla_{n} W$ and $\nabla^{\hat{\phi}} W=\hat{\nabla} W$ is, by (5.8),

$$
\left(\hat{\nabla}-\nabla_{n}\right) W=\frac{\cos f_{\hat{\mu}}-\cos f_{\mu_{n}}}{\sin \theta}\left(\mathcal{V} e_{2} \otimes \hat{E}_{2}-\mathcal{U} e_{2} \otimes \hat{E}_{1}\right)=\frac{\cos f_{\hat{\mu}}-\cos f_{\mu_{n}}}{\sin \theta} J^{N} W .
$$

An elementary calculation shows that

$$
\begin{aligned}
\frac{\cos f_{\hat{\mu}}-\cos f_{\mu_{n}}}{\sin \theta} & =\frac{\left(\hat{\mu}^{2}-\mu_{n}^{2}\right) \sin 2 \theta}{2\left(\hat{\mu}^{2} \cos ^{2} \frac{\theta}{2}+\sin ^{2} \frac{\theta}{2}\right)\left(\mu_{n}^{2} \cos ^{2} \frac{\theta}{2}+\sin ^{2} \frac{\theta}{2}\right)} \\
\Rightarrow\left|\frac{\cos f_{\hat{\mu}}-\cos f_{\mu_{n}}}{\sin \theta}\right| & \leq \frac{1}{2}\left(1+\frac{1}{\hat{\mu}^{2}}+\frac{1}{\mu_{n}^{2}}+\frac{1}{\hat{\mu}^{2} \mu_{n}^{2}}\right)\left|\hat{\mu}^{2}-\mu_{n}^{2}\right| .
\end{aligned}
$$

Hence, we have a pointwise bound of the form (4.1),

$$
\left|\left(\hat{\nabla}-\nabla_{n}\right) W\right| \leq a_{n}|W|
$$


where

$$
a_{n}=\frac{1}{2}\left(1+\frac{1}{\hat{\mu}^{2}}+\frac{1}{\mu_{n}^{2}}+\frac{1}{\hat{\mu}^{2} \mu_{n}^{2}}\right)\left|\hat{\mu}^{2}-\mu_{n}^{2}\right| \rightarrow 0
$$

as $n \rightarrow \infty$, as required.

Having shown that $\tau(\mu)$ is continuous, we now address its behaviour as $\mu \rightarrow \infty$. In particular, we will prove that $\lim _{\mu \rightarrow \infty} \tau(\mu)=0$. To do this, it suffices to consider only sections of $\phi^{*} T N$ of a certain type, which we will call "irrotational," namely those of the form

$$
V=\mathcal{V}(\theta) \widetilde{E}_{1}
$$

It is straightforward to compute $\mathfrak{D}^{\phi} V$ for such sections, and hence obtain $\operatorname{Hess}_{\phi}(V, V)$ as an explicit integral functional of $\mathcal{V}$. It follows from (5.8) that

$$
\mathfrak{D}^{\phi} V=-\left(\mathcal{V}^{\prime}-\frac{\cos f_{\mu}}{\sin \theta} \mathcal{V}\right)\left(e_{1} \otimes \widetilde{E}_{2}+e_{2} \otimes \widetilde{E}_{1}\right)
$$

The Hessian for irrotational sections is

$$
\operatorname{Hess}_{\phi}(V, V)=\frac{1}{2}\left\|\mathfrak{D}^{\phi} V\right\|_{L^{2}}^{2}=2 \pi \int_{0}^{\pi} d \theta \sin \theta\left(\mathcal{V}^{\prime}-\frac{\cos f_{\mu}}{\sin \theta} \mathcal{V}\right)^{2}
$$

We seek to compare this quantity, for irrotational sections $L^{2}$ orthogonal to ker $\mathfrak{D}^{\phi}$, with $\|V\|_{H^{1}}^{2}$. Note that ker $\mathfrak{D}^{\phi}$ is six-dimensional, and is spanned by

$$
K_{\mu}^{m}=-\frac{2 \cot ^{m}(\theta / 2)}{1+\mu^{2} \cot ^{2}(\theta / 2)}\left[\cos (1-m) \varphi \widetilde{E}_{1}+\sin (1-m) \varphi \widetilde{E}_{2}\right], \quad m=-1,0,1
$$

and their images under $J^{N}$. This basis is obtained by considering curves in $\mathrm{M}_{1}$ through $\phi$ generated by altering the real part of one of the coefficients of the rational map. Every irrotational section $V$ is automatically $L^{2}$ orthogonal to $K_{\mu}^{-1}, K_{\mu}^{0}, J^{N} K_{\mu}^{-1}, J^{N} K_{\mu}^{0}$ and $J^{N} K_{\mu}^{1}$, so we need only insist that $V$ is $L^{2}$ orthogonal to the one and only irrotational section in the basis, $K_{\mu}^{1}$, which we will henceforth denote $K_{\mu}$. Explicitly, we require that

$$
\left\langle V, K_{\mu}\right\rangle_{L^{2}}=-2 \pi \int_{0}^{\pi} d \theta \sin \theta \frac{\mathcal{V}(\theta) \sin \theta}{\sin ^{2} \frac{\theta}{2}+\mu^{2} \cos ^{2} \frac{\theta}{2}}=0
$$

having rearranged $K_{\mu}$ slightly.

We shall also need an explict formula for $\|V\|_{H_{1}}$. Equation (5.8) implies that

$$
\nabla^{\phi} V=\mathcal{V}^{\prime} e_{1} \otimes \widetilde{E}_{1}+\mathcal{V} \frac{\cos f_{\mu}}{\sin \theta} e_{2} \otimes \widetilde{E}_{2}
$$

So

$$
\begin{aligned}
\|V\|_{H^{1}}^{2} & =\left\|\nabla^{\phi} V\right\|_{L^{2}}^{2}+\|V\|_{L^{2}}^{2}=2 \pi \int_{0}^{\pi} d \theta \sin \theta\left(\left(\mathcal{V}^{\prime}\right)^{2}+\frac{\cos ^{2} f_{\mu}}{\sin ^{2} \theta} \mathcal{V}^{2}+\mathcal{V}^{2}\right) \\
& =\operatorname{Hess}_{\phi}(V, V)+\|V\|_{L^{2}}^{2}+2 \pi \int_{0}^{\pi} d \theta 2 \mathcal{V} \mathcal{V}^{\prime} \cos f_{\mu} \\
& =\operatorname{Hess}_{\phi}(V, V)+\|V\|_{L^{2}}^{2}+\left\langle V, \mathcal{E}_{\mu} V\right\rangle_{L^{2}}
\end{aligned}
$$


where $\mathcal{E}_{\mu} \in C^{\infty}\left(S^{2}\right)$ is the energy density of the map $\phi$. To see the last equality, note that $\phi$ is holomorphic, hence conformal, so

$$
f_{\mu}^{\prime}=\left|d \phi E_{1}\right|=\left|d \phi E_{2}\right|=\frac{\sin f_{\mu}}{\sin \theta} .
$$

Equation (5.19) follows from (5.20) and integration by parts.

It also proves useful to write down the formulae above using cylindrical coordinates instead of spherical polar coordinates on the domain. That is in place of $(\theta, \varphi)$ we use $(s, \varphi)$ where $s:=\log \cot \theta / 2$. First notice, that with respect to these cylindrical coordinates $f_{\mu}(s)$ arises by translating one fixed profile by a $\mu$ dependent amount. More precisely,

$$
f_{\mu}(s)=f_{1}(s+\log \mu)
$$

where

$$
f_{1}(s)=2 \cot ^{-1}(\exp s) .
$$

We have an analogous formula for $K_{\mu}$ the irrotational part of the kernel of $\mathfrak{D}^{\phi}$

$$
K_{\mu}(s)=\operatorname{sech}(s+\log \mu) \widetilde{E}_{1} .
$$

It is a routine computation to obtain the following formulae for $\operatorname{Hess}_{\phi_{\mu}}(V, V),\left\langle V, \mathcal{E}_{\mu} V\right\rangle_{L^{2}}$ and the $L^{2}, H^{1}$ norms of an irrotational section $V$ from the corresponding formulae using spherical polars already presented

$$
\begin{gathered}
\langle V, V\rangle_{L^{2}}=\int_{-\infty}^{\infty} d s \operatorname{sech}^{2} s \mathcal{V}^{2} \\
\left\langle V, \mathcal{E}_{\mu} V\right\rangle_{L^{2}}=\int_{-\infty}^{\infty} d s \operatorname{sech}^{2}(s+\log \mu) \mathcal{V}^{2} \\
\langle V, V\rangle_{H^{1}}=\int_{-\infty}^{\infty} d s\left(\left(\frac{d \mathcal{V}}{d s}\right)^{2}+\tanh ^{2}(s+\log \mu) \mathcal{V}^{2}+\operatorname{sech}^{2} s \mathcal{V}^{2}\right) \\
\operatorname{Hess}_{\phi_{\mu}}(V, V)=\int_{-\infty}^{\infty} d s\left(\frac{d \mathcal{V}}{d s}+\tanh (s+\log \mu) \mathcal{V}\right)^{2} .
\end{gathered}
$$

We may now state and prove the main result of this section. We shall denote the map $z \mapsto \mu z$ by $\phi_{\mu}$ to emphasize its parametric dependence.

Theorem 7 (global coercivity of the Hessian fails on $\operatorname{Rat}_{1}$ ) $\lim _{\mu \rightarrow \infty} \tau\left(\phi_{\mu}\right)=0$.

Proof: Define the following sequence of smooth irrotational sections $V_{\mu}=\mathcal{V}_{\mu} \widetilde{E}_{1}$ of $\phi_{\mu}^{*} T S^{2}$ where

$$
\mathcal{V}_{\mu}(s)=\operatorname{sech} s-c_{\mu} K_{\mu}(s)
$$

and $c_{\mu}$ is a constant determined by the requirement that $\left\langle V_{\mu}, K_{\mu}\right\rangle_{L^{2}}=0$. By our previous remarks, this suffices to ensure that $\mathcal{V}_{\mu}$ is orthogonal to $\operatorname{ker} \mathfrak{D}^{\phi_{\mu}}$. Since $V_{\mu} \in H^{1}\left(\phi_{\mu}^{*} T S^{2}\right) \cap$ ker $\mathfrak{D}^{\phi_{\mu}}$ it suffices to prove that

$$
\lim _{\mu \rightarrow \infty} \frac{\operatorname{Hess}_{\phi_{\mu}}\left(V_{\mu}, V_{\mu}\right)}{\left\langle V_{\mu}, V_{\mu}\right\rangle_{H^{1}}^{2}}=0 .
$$


In fact, we will show that

$$
\lim _{\mu \rightarrow \infty} \frac{\operatorname{Hess}_{\phi_{\mu}}\left(V_{\mu}, V_{\mu}\right)}{\left\langle V_{\mu}, \mathcal{E}_{\mu} V_{\mu}\right\rangle_{L^{2}}}=0
$$

which implies (5.29) since

$$
\left.\|V\|_{H^{1}}^{2}=\operatorname{Hess}_{\phi_{\mu}}(V, V)+\|V\|_{L^{2}}^{2}+\left\langle V, \mathcal{E}_{\mu} V\right\rangle_{L^{2}}\right\rangle\left\langle V, \mathcal{E}_{\mu} V\right\rangle_{L^{2}} .
$$

To prove (5.30), first notice that since $K_{\mu} \in \operatorname{ker} \mathfrak{D}^{\phi_{\mu}}$ we have

$$
\operatorname{Hess}_{\phi_{\mu}}\left(V_{\mu}, V_{\mu}\right)=\operatorname{Hess}_{\phi_{\mu}}(\operatorname{sech} s, \operatorname{sech} s) .
$$

Using the explicit expression for the Hessian in cylindrical coordinates we find

$$
\operatorname{Hess}_{\phi_{\mu}}(\operatorname{sech} s, \operatorname{sech} s)=\int_{-\infty}^{\infty} d s \operatorname{sech}^{2} s\left(\tanh s_{\mu}-\tanh s\right)^{2}
$$

where $s_{\mu}:=s+\log \mu$. Since $\left|\left(\tanh s_{\mu}-\tanh s\right)^{2}\right|<4$ holds for all $s$,

$$
\operatorname{Hess}_{\phi_{\mu}}(\operatorname{sech} s, \operatorname{sech} s)<4 \int_{-\infty}^{\infty} d s \operatorname{sech}^{2} s=8
$$

holds for all $\mu$. Since $\operatorname{Hess}_{\phi_{\mu}}\left(V_{\mu}, V_{\mu}\right)$ is bounded for all $\mu$, (5.30) is implied by

$$
\lim _{\mu \rightarrow \infty}\left\langle V_{\mu}, \mathcal{E}_{\mu} V_{\mu}\right\rangle_{L^{2}}=+\infty .
$$

To establish (5.31), first introduce for each nonnegative pair of integers $(m, n)$ the positive function

$$
I_{m, n}(\mu)=\int_{-\infty}^{\infty} d s \operatorname{sech}^{m} s \operatorname{sech}^{n} s_{\mu} .
$$

Clearly, the function $I_{m, n}(\mu)$ is bounded above by the constant $I_{m, 0}$. From the definition of $c_{\mu}$ we have

$$
c_{\mu}=\frac{\left\langle\text { sech }, K_{\mu}\right\rangle}{\left\langle K_{\mu}, K_{\mu}\right\rangle}=\frac{I_{3,1}}{I_{2,2}},
$$

and from equation (5.25) and the definition of $V_{\mu}$ we have

$$
\left\langle V_{\mu}, \mathcal{E}_{\mu} V_{\mu}\right\rangle_{L^{2}}=c_{\mu}\left(c_{\mu} I_{4,0}-2 I_{1,3}\right)+I_{2,2} .
$$

Since $I_{4,0}$ is a positive constant and $I_{1,3}, I_{2,2}$ are positive and bounded above, (5.31) will follow if we can establish that $\lim _{\mu \rightarrow \infty} c_{\mu}=+\infty$. In fact, one can explicitly evaluate the integrals which appear in the definition of $c_{\mu}$ to obtain

$$
c_{\mu}=\frac{I_{3,1}}{I_{2,2}}=\frac{\mu^{4}-4 \mu^{2} \log \mu-1}{4 \mu\left(\mu^{2} \log \mu-\mu^{2}+\log \mu+1\right)} .
$$

Clearly, this implies that $\lim _{\mu \rightarrow \infty} c_{\mu}=+\infty$ as required. 


\section{The case of $\operatorname{Rat}_{n}^{e q}$}

Staying in the context of the $\mathbb{C} P^{1}$ model on $S^{2}$, but generalizing to any degree sector, $n \geq 1$, things become rather more difficult. The reason is that the isometric action of $G$ on $\mathrm{M}_{n}=\operatorname{Rat}_{n}$ does not have cohomogeneity 1 , except for $n=1$. In general, then, to understand the global behaviour of $\tau$ one must consider the $4(n-1)$ dimensional orbifold $\mathrm{M}_{n} / G$, not simply a curve, as in section 5. Reduction to a curve is possible, however, provided we restrict attention to dynamics within a certain equivariance class. Employing stereographic coordinates $z, W$ on domain and codomain as usual, and defining polar coordinates such that $z=r e^{i \varphi}$, one may write the field equation for the $\mathbb{C} P^{1}$ model on spacetime $S^{2} \times \mathbb{R}$ as

$$
\frac{W_{t t}}{\left(1+r^{2}\right)^{2}}-\left(W_{r r}+\frac{1}{r} W_{r}+\frac{1}{r^{2}} W_{\varphi \varphi}\right)-\frac{2 \bar{W}}{1+|W|^{2}}\left(\frac{W_{t}^{2}}{\left(1+r^{2}\right)^{2}}-W_{r}^{2}-\frac{1}{r^{2}} W_{\varphi}^{2}\right)=0 .
$$

This supports equivariant solutions within the ansatz

$$
W(r, \varphi, t)=r^{n} q(r, t) e^{i n \varphi} .
$$

Provided $q:[0, \infty) \times(-\epsilon, \epsilon) \rightarrow \mathbb{C}$ is nowhere vanishing and has suitable boundary behaviour, solutions within this ansatz have degree $n$. Substituting (6.2) into (6.1) one obtains a $(1+1)$ dimensional hyperbolic partial differential equation for $q$. The space of static solutions is simply $q(r, t)=c \neq 0$, a complex constant. So the equivariant moduli space is $\mathrm{M}_{n}^{e q}=\operatorname{Rat}_{n}^{e q}=$ $\left\{z \mapsto c z^{n}: c \neq 0\right\} \cong \mathbb{C}^{\times}$, the punctured complex plane. Note that $\mathrm{M}_{n}^{e q}$ is one connected component of the fixed point set in $\mathrm{M}_{n}$ of the isometry group $G^{e q}=\left\{\left(\left[\exp -i n \frac{\psi}{2} \tau_{3}\right]\right.\right.$, $\left.\left[\exp i \frac{\psi}{2} \tau_{3}\right]\right)$ : $\psi \in \mathbb{R}\} \cong S O(2)$, so we are assured that $\mathrm{M}_{n}^{e q} \subset \mathrm{M}_{n}$ is totally geodesic. Both the true wave map flow and its geodesic approximant stay within the equivariance class, therefore, and one can again ask to what extent the analytic method of Stuart applies. The equivariant configuration space enjoys a residual $U(1)$ symmetry, namely $W(z) \mapsto e^{i \psi} W(z)$, and the discrete symmetry $W(z) \mapsto 1 / W(1 / z)$, both of which leave the harmonic map energy unchanged, so we may again restrict attention to a curve in $\mathrm{M}_{n}^{e q}$, namely $\Gamma=\left\{z \mapsto \mu z^{n}: \mu \in[1, \infty)\right\}$. The situation is actually simpler than in the full degree 1 case, because now the error, that is, the section of $\phi^{*} T N$ along which we exponentiate to get from the holomorphic approximant to the true solution at time $t$, must also lie within the equivariance class. The only sections of relevance, therefore, are irrotational sections and their $J^{N}$ images. We may define a new optimal constant $\tau^{e q}(\phi)$, again the infimium of the Hessian for $H^{1}$ unit sections $L^{2}$ orthogonal to ker $\mathfrak{D}^{\phi}$, but now we include only equivariant sections. Since these form a subset of all sections, we have trivially that $\tau^{e q}(\phi) \geq \tau(\phi)>0$ by Theorem 3 ,

Once again, we may think of $\tau^{e q}$ as a function of $\mu \in[1, \infty)$. We first show that $\tau^{e q}(\mu)$ is continuous. Since the setup is very similar to section [5] we use equivalent notation and conventions, and omit several details. In spherical polar coordinates, the map $\phi: z \mapsto \mu z^{n}$ is

$$
(\theta, \varphi) \mapsto\left(f_{\mu}(\theta), n \varphi\right), \quad f_{\mu}(\theta)=2 \cot ^{-1}\left(\mu \cot ^{n} \frac{\theta}{2}\right)
$$

so $d \phi E_{1}=f_{\mu}^{\prime} \widetilde{E}_{1}$ and $d \phi E_{2}=n \operatorname{cosec} \theta \cos f_{\mu} \widetilde{E}_{2}$. It follows that

$$
\nabla^{\phi} \widetilde{E}_{1}=n \frac{\cos f_{\mu}}{\sin \theta} e_{2} \otimes \widetilde{E_{2}}, \quad \nabla^{\phi} \widetilde{E}_{2}=-n \frac{\cos f_{\mu}}{\sin \theta} e_{2} \otimes \widetilde{E_{1}}
$$


Hence, on an irrotational section $V=\mathcal{V}(\theta) \widetilde{E}_{1}$,

$$
\nabla^{\phi} V=V^{\prime} e_{1} \otimes \widetilde{E}_{1}+V n \frac{\cos f_{\mu}}{\sin \theta} e_{2} \otimes \widetilde{E}_{2}
$$

Given a general equivariant section $W=V+J^{N} U$, where $V, U$ are irrotational, we may transfer $W$ to a neighbouring bundle $\hat{\phi}^{*} T N$ (where $\hat{\phi}: z \mapsto \hat{\mu} z$ ) using the canonical isometry (5.2), act with $\nabla^{\widehat{\phi}}$, then transfer back again, to obtain $\hat{\nabla} W$. The difference between this and $\nabla^{\phi} W$ is

$$
\left(\nabla^{\phi}-\hat{\nabla}\right) W=n \frac{\cos f_{\mu}-\cos f_{\hat{\mu}}}{\sin \theta} J^{N} W
$$

Now

$$
\begin{aligned}
\alpha(\theta)=\frac{\cos f_{\mu}-\cos f_{\hat{\mu}}}{\sin \theta} & =\frac{\left(\mu^{2}-\hat{\mu}^{2}\right)(\cos (\theta / 2) \sin (\theta / 2))^{2 n-1}}{\left(\sin ^{2 n}(\theta / 2)+\mu^{2} \cos ^{2 n}(\theta / 2)\right)\left(\sin ^{2 n}(\theta / 2)+\hat{\mu}^{2} \cos ^{2 n}(\theta / 2)\right)} \\
\Rightarrow|\alpha(\theta)| & \leq\left(1+\frac{1}{\hat{\mu}^{2}}+\frac{1}{\mu^{2}}+\frac{1}{\hat{\mu}^{2} \mu^{2}}\right)\left|\hat{\mu}^{2}-\mu^{2}\right| .
\end{aligned}
$$

So the pullback connexion satisfies a pointwise bound of the correct type $\left(\left|\left(\nabla^{\phi}-\hat{\nabla}\right) W\right|<\right.$ $c(\mu, \hat{\mu})|W|$ where $c \rightarrow 0$ as $\mu \rightarrow \hat{\mu})$ and we may conclude from the results of section 4 that $\tau^{e q}(\mu)$ is continuous.

As for Rat $_{1}$, the interesting issue is the large $\mu$ behaviour of $\tau^{e q}(\mu)$. We are so far unable to prove anything rigorous about $\lim _{\mu \rightarrow \infty} \tau^{e q}(\mu)$ (if, indeed, it exists). However, we make the following conjecture:

Conjecture 8 For all $n \geq 1, \lim _{\mu \rightarrow \infty} \tau^{e q}(\mu)$ exists and is finite. For all $n>1$, this limit is not zero.

If true, the equivariant Hessian is globally coercive for degree $n$ greater than unity. One could at least hope, therefore, to model equivariant lump collapse accurately within the geodesic approximation in the case $n>1$.

To motivate this conjecture, we should describe some numerical work which led directly to the proof of its $n=1$ counterpart, Theorem [7. It is straightforward to write the Jacobi operator on irrotational sections as an explicit ordinary differential operator acting on $\mathcal{V}(\theta)$. The eigenvalue problem for $\mathfrak{J}^{\phi}$ then reduces to a singular Sturm-Liouville problem on $(0, \pi)$, namely

$$
-\mathcal{V}^{\prime \prime}-\cot \theta \mathcal{V}^{\prime}+n^{2} \frac{\cos 2 f_{\mu}}{\sin ^{2} \theta} \mathcal{V}=\omega^{2} \mathcal{V}
$$

Here $\omega^{2} \geq 0$ is the eigenvalue. The eigenfunctions of this problem form an $L^{2}$ orthogonal basis for the space of irrotational sections. In particular, the eigenfunctions outside the kernel, which we shall refer to as "excited states", in analogy with quantum mechanics, form a basis for the irrotational sections orthogonal to ker $\mathfrak{J}^{\phi}$. If we could prove that $\operatorname{Hess}_{\phi}(V, V) /\|V\|_{H^{1}}^{2}=\omega^{2}\|V\|_{L^{2}}^{2} /\|V\|_{H^{1}}^{2}$ is bounded uniformly away from zero for all $\mu$ and all excited states $V$, global coercivity of Hess $^{e q}$ would follow. Conversely, if for one of the excited states, $\omega^{2}\|V\|_{L^{2}}^{2} /\|V\|_{H^{1}}^{2} \rightarrow 0$ as $\mu \rightarrow \infty$, global coercivity must fail: the one parameter family 


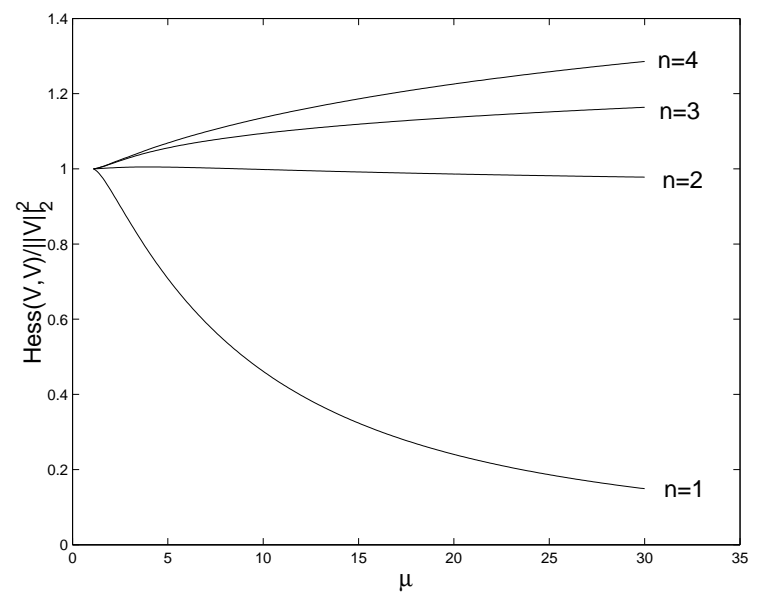

Figure 1: Plots of the ratio Hess $_{\phi_{\mu}}\left(V_{\mu}, V_{\mu}\right) /\left\|V_{\mu}\right\|_{H^{1}}^{2}$ against $\mu$ for the first excited state $V_{\mu}$ of the Sturm-Liouville problem (6.8) with $\phi_{\mu}: z \rightarrow \mu z^{n}, n=1,2,3,4$. To facilitate comparison of the curves, in each case the ratio has been normalized by its value at $\mu=1(0.30,0.74,1.17$ and 1.55 respectively, to 2 decimal places). Note that only for $n=1$ does the ratio tend to 0 as $\mu \rightarrow \infty$.

of excited states itself provides a counterexample. Unfortunately, the ( $\mu$ families) of eigensections are rather inaccessible analytically (except in the special case $\mu=n=1$, where they are known exactly). However, they are quite easy to construct numerically, either by employing a specialist Sturm-Liouville solver package, or by using a shooting method. We have tried both strategies, obtaining compatible results from each.

The pertinent results may be summarized as follows. For $n=1,2,3,4$ and for all excited states from 2nd to 6 th (ordered by increasing $\omega^{2}$ ), the $\operatorname{ratio}_{\operatorname{Hess}_{\phi}}(V, V) /\|V\|_{H_{1}}^{2}$ appears to remain bounded away from zero as $\mu \rightarrow \infty$. More interesting is the first excited state. Here the ratio remains bounded away from zero for $n=2,3,4$, but not for $n=1$. It was by examining the graphs of the $n=1$ first excited eigenstates that the explicit family $V_{\mu}$ of the proof of Theorem 7 was devised: the family is designed to have the same qualitative behaviour as the numerically generated eigenstates. In figure 1 we present a graph showing the ratio $\operatorname{Hess}_{\phi}(V, V) /\|V\|_{H_{1}}^{2}$ as a function of $\mu$ for the first excited states for $n=1,2,3,4$. These data were generated by a shooting method using a 4th order Runge-Kutta scheme with variable $\theta$ step. The singularities at $\theta=0, \pi$ were handled by series expansions, so the scheme shot forwards from $\theta=\delta$ and backwards from $\theta=\pi-\delta$, applying a matching condition at $\theta=\frac{\pi}{2}(\delta$ being a small positive number, typically 0.001). The difference between $n=1$ and the other cases is quite clear. Although it is impossible to be exhaustive numerically, the results suggest that $\operatorname{Hess}_{\phi}(V, V) /\|V\|_{H_{1}}^{2}$ is bounded away from zero for $n>1$, as we have conjectured. Linhart and Sadun in a recent numerical study of lump collapse in the $\mathbb{C} P^{1}$ model on $\mathbb{C}$ imposed the analogous equivariance condition on their field equation [10]. It is an intriguing coincidence that they found that single lump collapse differs greatly from the predictions of the geodesic approximation (truncated to a finite disk in $\mathbb{C}$ ), whereas the collapse of two coincident lumps is quite well modelled by the geodesic flow. 


\section{References}

[1] D. Adams and L. Simon, "Rates of asymptotic convergence near isolated singularities of geometric extrema" Indiana J. Math. 37 (1988) 225-54.

[2] M.F. Atiyah and N.J. Hitchin, The Geometry and Dynamics of Magnetic Monopoles (Princeton University Press, Princeton, USA, 1988).

[3] P. Bizon, T. Chmaj and Z. Tabor, "Formation of singularities for equivariant $(2+1)$ dimensional wave maps into the 2-sphere" Nonlinearity 14 (2001) 1041-53.

[4] Y. Choquet-Bruhat, "Global existence of wave maps" Rendiconti del Circolo Matematico di Palermo, Serie II Suppl. 57 (1998) 143-52.

[5] G.W. Gibbons and N.S. Manton, "Classical and quantum dynamics of BPS monopoles" Nucl. Phys. B274 (1986) 183-224.

[6] A. Hatcher, Algebraic Topology (Cambridge University Press, Cambridge, UK, 2002) p8.

[7] R.A. Leese, "Low energy scattering of solitons in the $\mathbb{C} P^{1}$ model" Nucl. Phys. B344 (1990) 33-72.

[8] L. Lemaire and J.C. Wood, "Jacobi fields along harmonic 2-spheres in $\mathbb{C} P^{2}$ are integrable" J. London Math. Soc. 66 (2002) 329-40.

[9] A. Lichnerowicz, "Applications harmoniques et variétés kähleriennes" Symp. Math. Bologna 3 (1970) 341-402.

[10] J.M. Linhart and L.A. Sadun, "Fast and slow blowup in the $S^{2} \sigma$ model and the $(4+1)$ dimensional Yang-Mills model" Nonlinearity 15 (2002) 219-38.

[11] N.S. Manton, "A remark on the scattering of BPS monopoles" Phys. Lett. $110 B$ (1982) 54-6.

[12] N.S. Manton and S.M. Nasir, "Volume of vortex moduli spaces" Commun. Math. Phys. 199 (1999) 591-604.

[13] D. McDuff and D. Salamon, J-holomorphic curves and quantum cohomology (American Mathematical Society, Providence, RI, USA 1994) p182.

[14] R.S. Palais, Foundations of global non-linear analysis (W. A. Benjamin, Inc., New YorkAmsterdam, 1968).

[15] P.J. Ruback, "Sigma model solitons and their moduli space metrics" Commun. Math. Phys. 116 645-58.

[16] L. Sadun and J.M. Speight, "Geodesic incompleteness in the $\mathbb{C} P^{1}$ model on a compact Riemann surface" Lett. Math. Phys. 43 (1998) 329-34.

[17] T.M. Samols, "Vortex scattering" Commun. Math. Phys. 145 (1992) 149-79. 
[18] J. Shatah and M. Struwe, Geometric Wave Equations (American Mathematical Society, Providence RI, USA, 1998).

[19] J.M. Speight, "Low energy dynamics of a $\mathbb{C} P^{1}$ lump on the sphere" J. Math. Phys. 36 (1995) 796-813.

[20] J.M. Speight, "Lump dynamics in the $\mathbb{C} P^{1}$ model on the torus" Commun. Math. Phys. 194 (1998) 513-39.

[21] J.M. Speight, "The $L^{2}$ geometry of spaces of harmonic maps $S^{2} \rightarrow S^{2}$ and $\mathbb{R} P^{2} \rightarrow \mathbb{R} P^{2}$ " to appear in J. Geom. Phys.

[22] I. Stokoe and W.J. Zakrzewski, "Dynamics of solutions of the $\mathbb{C} P^{N-1}$ models in $(2+1)$ dimensions" Z. Phys. C - Particles and Fields 34 (1987) 491-6.

[23] D. Stuart, "Dynamics of abelian Higgs vortices in the near Bogomolny regime" Commun. Math. Phys. 159 (1994) 51-91.

[24] D. Stuart, "The geodesic approximation for the Yang-Mills-Higgs equations" Commun. Math. Phys. 166 (1994) 149-90.

[25] H. Urakawa, Calculus of Variations and Harmonic Maps (American Mathematical Society, Providence RI, USA, 1993) chap. 5.

[26] R.S. Ward, "Slowly moving lumps in the $\mathbb{C} P^{1}$ model in $(2+1)$ dimensions" Phys. Lett. 158B (1985) 424-8.

[27] T.J. Willmore, Riemannian Geometry (Clarendon Press, Oxford, UK, 1993) p57. 